\title{
A Framework for Coxeter Spectral Classification of Finite Posets and Their Mesh Geometries of Roots
}

\author{
Daniel Simson and Katarzyna Zając \\ Faculty of Mathematics and Computer Science, Nicolaus Copernicus University, Ulica Chopina 12/18, 87-100 Toruń, Poland \\ Correspondence should be addressed to Daniel Simson; simson@mat.umk.pl
}

Received 28 March 2012; Accepted 1 November 2012

Academic Editor: Marco Squassina

Copyright (C) 2013 D. Simson and K. Zając. This is an open access article distributed under the Creative Commons Attribution License, which permits unrestricted use, distribution, and reproduction in any medium, provided the original work is properly cited.

\begin{abstract}
Following our paper [Linear Algebra Appl. 433(2010), 699-717], we present a framework and computational tools for the Coxeter spectral classification of finite posets $J \equiv(J, \preceq)$. One of the main motivations for the study is an application of matrix representations of posets in representation theory explained by Drozd [Funct. Anal. Appl. 8(1974), 219-225]. We are mainly interested in a Coxeter spectral classification of posets $J$ such that the symmetric Gram matrix $G_{J}:=(1 / 2)\left[C_{J}+C_{J}^{\text {tr }}\right] \in \mathbb{M}_{J}(\mathbb{Q})$ is positive semidefinite, where $C_{J} \in \mathbb{M}_{J}(\mathbb{Z})$ is the incidence matrix of $J$. Following the idea of Drozd mentioned earlier, we associate to $J$ its Coxeter matrix $\operatorname{Cox}_{J}:=-C_{J} \cdot C_{J}^{-\operatorname{tr}}$, its Coxeter spectrum specc ${ }_{J}$, a Coxeter polynomial $\operatorname{cox}_{J}(t) \in \mathbb{Z}[t]$, and a Coxeter number $\mathbf{c}_{J}$. In case $G_{J}$ is positive semi-definite, we also associate to $J$ a reduced Coxeter number $\check{\mathbf{c}}_{J}$, and the defect homomorphism $\partial_{J}: \mathbb{Z}^{J} \rightarrow \mathbb{Z}$. In this case, the Coxeter spectrum specc $_{J}$ is a subset of the unit circle and consists of roots of unity. In case $G_{J}$ is positive semi-definite of corank one, we relate the Coxeter spectral properties of the posets $J$ with the Coxeter spectral properties of a simply laced Euclidean diagram $D J \in\left\{\widetilde{\mathbb{D}}_{n}, \widetilde{\mathbb{E}}_{6}, \widetilde{\mathbb{E}}_{7}, \widetilde{\mathbb{E}}_{8}\right\}$ associated with $J$. Our aim of the Coxeter spectral analysis of such posets $J$ is to answer the question when the Coxeter type $\mathbf{C t y p e}_{J}:=\left(\operatorname{specc}_{J}, \mathbf{c}_{J}, \check{\mathbf{c}}_{J}\right)$ of $J$ determines its incidence matrix $C_{J}$ (and, hence, the poset $J$ ) uniquely, up to a $\mathbb{Z}$-congruency. In connection with this question, we also discuss the problem studied by Horn and Sergeichuk [Linear Algebra Appl. 389(2004), 347-353], if for any $\mathbb{Z}$-invertible matrix $A \in \mathbb{M}_{n}(\mathbb{Z})$, there is $B \in \mathbb{M}_{n}(\mathbb{Z})$ such that $A^{\text {tr }}=B^{\operatorname{tr}} \cdot A \cdot B$ and $B^{2}=E$ is the identity matrix.
\end{abstract}

\section{Introduction}

In the present paper, we continue our Coxeter spectral study of finite posets, started in [1], in a close connection with the Coxeter spectral technique introduced in [2-4] for acyclic edge-bipartite graphs or signed graphs in the sense of [5]. We also follow some of the techniques of representation theory, graph combinatorics, and the spectral graph theory; see [631].

Here, we use the terminology and notation introduced in $[1,4,26-28]$. We denote by $\mathbb{N} \subseteq \mathbb{Z} \subseteq \mathbb{Q}$ the set of nonnegative integers, the ring of integers, and the rational number field. Given $m \geq 1$, we view $\mathbb{Z}^{m}$ as a free abelian group and denote by $e_{1}, \ldots, e_{m}$ the standard $\mathbb{Z}$-basis of $\mathbb{Z}^{m}$. Given an index set $J$, we denote by $\mathbb{Z}^{J}$ the abelian group of all vectors $v=\left(v_{j}\right)_{j \in J}$, with integer coordinates $v_{j} \in \mathbb{Z}$, by $\mathbb{M}_{J}(\mathbb{Z})$ the $\mathbb{Z}$-algebra of all square $J$ by $J$ integral matrices, and by $E \in \mathbb{M}_{J}(\mathbb{Z})$ the identity matrix. In particular, $\mathbb{M}_{m}(\mathbb{Z})$, with $m \geq 1$, is the $\mathbb{Z}$-algebra of all square $m$ by $m$ matrices. The group

$$
\operatorname{Gl}(m, \mathbb{Z}):=\left\{A \in \mathbb{M}_{m}(\mathbb{Z}), \operatorname{det} A \in\{-1,1\}\right\} \subseteq \mathbb{M}_{m}(\mathbb{Z})
$$

is called the (integral) general linear group. We say that two square rational matrices $A, A^{\prime} \in \mathbb{M}_{m}(\mathbb{Q})$ are $\mathbb{Z}$-equivalent, or $\mathbb{Z}$-congruent, (and denote $A \sim_{\mathbb{Z}} A^{\prime}$ ) if there is a matrix $B \in$ $\mathrm{Gl}(m, \mathbb{Z})$ such that $A^{\prime}=B^{\text {tr }} \cdot A \cdot B$. By a poset $J \equiv(J, \preceq)$ we mean a finite partially ordered set $J$ with respect to a partial order relation $\preceq$. Following [26], a poset $J$ is called a one-peak poset if $J$ has a unique maximal element $*$. A finite poset $J$ is uniquely determined by its incidence matrix $C_{J} \in \mathbb{M}_{J}(\mathbb{Z})$, that is, the square $J \times J$ matrix, as follows:

$$
C_{J}=\left[c_{i j}\right]_{i, j \in J} \in \mathbb{M}_{J}(\mathbb{Z}), \quad \text { with } c_{i j}= \begin{cases}1, & \text { for } i \preceq j, \\ 0, & \text { for } i \npreceq j .\end{cases}
$$


Following an idea of Drozd [32] (developed in [27]), we have introduced in $[1,28]$ the Tits matrix $\widehat{C}_{J} \in \mathbb{M}_{J}(\mathbb{Z})$ of $J$ to be the integral matrix

$$
\widehat{C}_{J}=\left[\widehat{c}_{i j}\right]_{i, j \in J} \in \mathbb{M}_{J}(\mathbb{Z}),
$$

with $\widehat{c}_{i j}\left\{\begin{array}{l}1, \quad i=j \text { or } j \preceq i, i, j \notin \max J, \\ 0, \quad i, j \text { incomparable, or } i \preceq j \text { and } i, j \notin \max J, \\ -1, \quad \text { if } i \prec j \text { and } j \in \max J,\end{array}\right.$

where $\max J$ is the set of all maximal elements of $J$. Usually, we equip the elements of $J$ with a numbering; that is, $J$ is viewed as $J=\left\{a_{1}, \ldots, a_{m}\right\}, m=|J| \geq 1$. Throughout, we fix such a numbering and make the identifications $\mathbb{M}_{m}(\mathbb{Z}) \equiv$ $\mathbb{M}_{J}(\mathbb{Z})$ and $\mathbb{Z}^{m} \equiv \mathbb{Z}^{J}$. The incidence matrix $C_{J} \in \mathbb{M}_{m}(\mathbb{Z}) \equiv$ $\mathbb{M}_{J}(\mathbb{Z})$ and the Tits matrix $\widehat{C}_{J} \in \mathbb{M}_{m}(\mathbb{Z}) \equiv \mathbb{M}_{J}(\mathbb{Z})$ depend on the numbering of $a_{1}, \ldots, a_{m}$. Namely, if $J^{\prime}=\left\{a_{1}^{\prime}, \ldots, a_{m}^{\prime}\right\}$ is obtained from $J=\left\{a_{1}, \ldots, a_{m}\right\}$ by a permutation $\sigma \in \mathbf{S}_{m}$ and $\widehat{\sigma} \in \operatorname{Gl}(m, \mathbb{Z})$ is the permutation matrix of $\sigma$, then

$$
C_{J^{\prime}}=\widehat{\sigma}^{-1} \cdot C_{J} \cdot \widehat{\sigma}, \quad \widehat{C}_{J^{\prime}}=\widehat{\sigma}^{-1} \cdot \widehat{C}_{J} \cdot \widehat{\sigma}
$$

Note that any poset $J$ admits an upper-triangular numbering $J=\left\{a_{1}, \ldots, a_{m}\right\}$; that is, $a_{i} \preceq a_{j}$ implies $i \leq j$. In this case, $C_{J} \in \mathbb{M}_{m}(\mathbb{Z})$ is an upper-triangular matrix with 1 on the main diagonal, and, hence, $\operatorname{det} C_{J}=1$, and $\operatorname{det} C_{J^{\prime}}=1$, for any numbering $J^{\prime}=\left\{a_{1}^{\prime}, \ldots, a_{m}^{\prime}\right\}$.

Fix a numbering $a_{1}, \ldots, a_{m}$ of elements of $J$. Following [1, 28 ], by the Euler matrix of the poset $J$ we mean the inverse

$$
\bar{C}_{J}:=C_{J}^{-1} \in \mathbb{M}_{m}(\mathbb{Z}) \equiv \mathbb{M}_{J}(\mathbb{Z})
$$

of $C_{J}$. Following $[3,4]$, we call

$$
\begin{gathered}
A d_{J}:=C_{J}+C_{J}^{\mathrm{tr}}-2 \cdot E, \\
P_{J}(t)=\operatorname{det}\left(t \cdot E-A d_{J}\right) \in \mathbb{Z}[t]
\end{gathered}
$$

the symmetric adjacency matrix and the characteristic polynomial of the poset $J$. The set $\operatorname{spec}_{J}$ of all $m=|J|$ real roots of $P_{J}(t)$ is defined to be the (real) spectrum of the poset $J$.

We denote by $q_{J}, \widehat{q}_{J}, \bar{q}_{J}: \mathbb{Z}^{J} \equiv \mathbb{Z}^{m} \rightarrow \mathbb{Z}$ the incidence quadratic form, the Tits quadratic form, and the Euler quadratic form of $J$ defined by the formulae

$$
\begin{gathered}
q_{J}(x)=x \cdot C_{J} \cdot x^{\operatorname{tr}}=\sum_{j \in J} x_{j}^{2}+\sum_{i<j} x_{i} x_{j}, \\
\widehat{q}_{J}(x)=x \cdot \widehat{C}_{J} \cdot x^{\operatorname{tr}}=\sum_{j \in J} x_{j}^{2}+\sum_{i<j \in J} x_{i} x_{j}-\sum_{p \in \max J} \sum_{i<p} x_{i} x_{p}, \\
\bar{q}_{J}(x)=x \cdot \bar{C}_{J} \cdot x^{\operatorname{tr}}=x \cdot C_{J}^{-1} \cdot x^{\operatorname{tr}},
\end{gathered}
$$

respectively, where $\check{J}=J \backslash \max J, \max J$ is the set of all maximal elements in $J$, and $\widehat{C}_{J} \in \mathbb{M}_{J}(\mathbb{Z})$ is the Tits matrix of $J$; see $(27)$ and $[1,28]$ for a definition. The matrices

$$
\begin{aligned}
& G_{J}:= \frac{1}{2}\left[C_{J}+C_{J}^{\mathrm{tr}}\right], \quad \widehat{G}_{J}:=\frac{1}{2}\left[\widehat{C}_{J}+\widehat{C}_{J}^{\mathrm{tr}}\right], \\
& \bar{G}_{J}:=\frac{1}{2}\left[\bar{C}_{J}+\bar{C}_{J}^{\mathrm{tr}}\right] \in \mathbb{M}_{J}(\mathbb{Q}),
\end{aligned}
$$

with rational coefficients, are called the symmetric incidence Gram matrix, the symmetric Tits-Gram matrix, and the symmetric Euler-Gram matrix of $J$. The matrices

$$
\begin{gathered}
\widehat{A} d_{J}:=\widehat{C}_{J}+\widehat{C}_{J}^{\operatorname{tr}}-2 \cdot E \\
\bar{A} d_{J}:=\bar{C}_{J}+\bar{C}_{J}^{\operatorname{tr}}-2 \cdot E=C_{J}^{-1}+C_{J}^{-\operatorname{tr}}-2 \cdot E
\end{gathered}
$$

with integer coefficients, are called the Tits adjacency matrix, and the Euler adjacency matrix of $J$. The polynomials

$$
\begin{gathered}
P_{J}(t):=\operatorname{det}\left(t \cdot E-A d_{J}\right)=\operatorname{det}\left(t \cdot E-\widehat{A} d_{J}\right), \\
\bar{P}_{J}(t):=\operatorname{det}\left(t \cdot E-\bar{A} d_{J}\right)
\end{gathered}
$$

are called the characteristic polynomial of $J$ and the Eulercharacteristic polynomial of $J$, respectively.

Example 1. (a) If $I$ is the poset

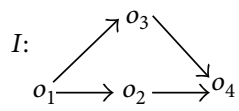

then $P_{I}(t)=\bar{P}_{I}(t)=t^{4}-5 t^{2}-4 t$; that is, the characteristic polynomial $P_{I}(t)$ of $I$ coincides with the Euler-characteristic polynomial $\bar{P}_{I}(t)$ of $I$.

(b) If $J$ is the poset

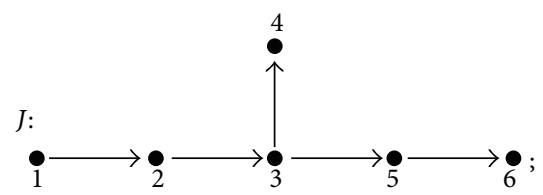

of the Dynkin type $\mathbb{E}_{6}$, then the characteristic polynomial $P_{J}(t)$ of $J$ does not coincide with the Euler-characteristic polynomial $\bar{P}_{J}(t)$ of $J$, because

$$
\begin{gathered}
P_{J}(t)=t^{6}-13 t^{4}-26 t^{3}-15 t^{2}+2 t+3, \\
\bar{P}_{J}(t)=t^{6}-5 t^{4}+5 t^{2}-1 .
\end{gathered}
$$

Following $[17,33]$, we introduce the following definition.

Definition 2. (a) We define a poset $J$ to be positive (resp., nonnegative) if the incidence form $q_{J}: \mathbb{Z}^{J} \rightarrow \mathbb{Z}$ of $J$ is positive (resp., nonnegative); that is, $q_{J}(v)>0$, for any nonzero $v \in \mathbb{Z}^{J}$ (resp., $q_{J}(v) \geq 0$, for any $\left.v \in \mathbb{Z}^{J}\right)$. 
(b) We define a poset $J$ to be principal if its incidence form $q_{J}: \mathbb{Z}^{J} \rightarrow \mathbb{Z}$ is principal in the sense of [34, Definition 2.1]; that is, $q_{J}$ is nonnegative, not positive, and the kernel

$$
\operatorname{Ker} q_{J}:=\left\{v \in \mathbb{Z}^{J} ; q_{J}(v)=0\right\}
$$

is an infinite cyclic subgroup of $\mathbb{Z}^{J}$.

Following the main idea of the Coxeter spectral analysis of acyclic edge-bipartite graphs (signed graphs) presented in $[3,4]$, we study finite posets $J$ (with a fixed numbering $J=$ $\left.\left\{a_{1}, \ldots, a_{m}\right\}\right)$ by means of the Coxeter spectrum

$$
\operatorname{specc}_{J} \subseteq \mathbb{C}
$$

of $J$, that is, the set $\operatorname{specc}_{J}$ of all $m=|J|$ eigenvalues of the Coxeter matrix

$$
\operatorname{Cox}_{J}:=-C_{J} \cdot C_{J}^{-\operatorname{tr}} \in \mathbb{M}_{m}(\mathbb{Z}) \equiv \mathbb{M}_{J}(\mathbb{Z})
$$

of $J$, or equivalently, the set specc $_{J}$ of all $m=|J|$ roots of the Coxeter polynomial

$$
\begin{aligned}
\operatorname{cox}_{J}(t) & :=\operatorname{det}\left(t \cdot E-\operatorname{Cox}_{J}\right)=\operatorname{det}\left(t \cdot E-\widehat{\operatorname{Cox}}_{J}\right) \\
& =\operatorname{det}(t \cdot E-\overline{\operatorname{Cox}}) \in \mathbb{Z}[t]
\end{aligned}
$$

see (31) and [1]. It follows from (4) that the Coxeter spectrum specc $_{J}$ of $J$ and the spectrum spec of $_{J}$ do not depend on the numbering of the elements of the poset $J$.

A motivation. We recall from $[26,27]$ that the problems we study in the paper have a bimodule matrix problem interpretation and have essential applications in reducing some classes of partitioned matrices with coefficients in a field $K$ to their canonical forms. For simplicity of its presentation, we illustrate it in case when $\widehat{q}_{J}(x)$ is the Tits quadratic form (7) of the poset $J=\left\{a_{1}, \ldots, a_{n}, *,+\right\}$, with an uppertriangular partial order $\preceq$ such that $J$ has precisely two maximal elements $*:=*_{n+1}$ and $+:=+_{n+2}$. In this case, we have

$$
\widehat{q}_{J}(x)=\sum_{a_{i} \in J} x_{i}^{2}+\sum_{a_{i}<a_{j}, i, j \leq n} x_{i} x_{j}-\sum_{a_{i}<*} x_{i} x_{*}-\sum_{a_{j}<+} x_{j} x_{+} .
$$

Fix a vector $v=\left(v_{1}, \ldots, v_{n}, v_{*}, v_{+}\right) \in \mathbb{N}^{n+2} \equiv \mathbb{N}^{J}$, and consider the $K$-vector space $\mathbf{M a t}_{v}^{J}$ of all partitioned matrices of the form (compare with [27])

$\left.A=\begin{array}{|c|c|c|c|}\hline A_{1 *} & A_{2 *} & \cdots & A_{n *} \\ \hline A_{1+} & A_{2+} & \cdots & A_{n+} \\ v_{v_{1}} & \cdots & \underbrace{}_{v_{n}}\end{array}\right\} v_{*}$

with coefficients in $K$, where $A_{i *}=0$ if $a_{i} k *$ and $A_{j+}=0$ if $a_{j} k+$. Consider the group $\mathbf{G}_{v}^{J}$ generated by the elementary transformations of the following three types: (a) all simultaneous transformations on rows inside each horizontal block;

(b) all simultaneous transformations on columns inside each vertical block;

(c) all simultaneous transformations on columns from the $i$ th column block to $j$ th column block, if the relation $a_{i} \preceq a_{j}$ holds in the poset $J \backslash\{*,+\}$ (with natural zero-adjustments).

It follows from [27, Section 2] (see also [16, 26, 32]) that the problem of finding canonical forms of matrices in $\mathbf{M a t}_{v}^{J}$, with respect to the elementary transformations from the set $\mathbf{G}_{v}^{J}$, is controlled by the Tits quadratic form $\hat{q}_{J}$ in the following sense. For any $v \in \mathbb{N}^{J}$, there is only a finite number $\mathbf{G}_{v}^{J}$-canonical forms of matrices in $\mathbf{M a t}_{v}^{J}$ if and only if the form $\hat{q}_{J}$ is weakly positive; that is, $\widehat{q}_{J}(v)$ is positive, for all nonzero vectors $v \in \mathbb{N}^{J}$. Moreover, there is one-to-one correspondence between the irreducible $\mathbf{G}_{v}^{J}$-canonical forms in $\mathbf{M a t}_{v}^{J}$ and the vectors $v \in \mathbb{N}^{J}$ satisfying the equation $\widehat{q}_{J}(v)=$ 1. A solution of the problem is given in [27] and [1, Theorem 1.6]. A useful homological interpretation (in terms of the Euler characteristic) of the $\mathbb{Z}$-bilinear Tits form $\widehat{b}_{J}(x, y)=$ $x \cdot \widehat{C}_{J} \cdot y^{\operatorname{tr}}(26)$ and $\mathbb{Z}$-bilinear Euler form $\bar{b}_{J}(x, y)=x \cdot \bar{C}_{J} \cdot y^{\operatorname{tr}}$ is given in $[1,(1.3)]$. The reader is referred to $[6-8,25]$ for a detailed study and a solution of other important matrix problems of high computational complexity that have many useful applications in representation theory; see $[16,26]$.

We show in Section 3 that the Coxeter spectral analysis of principal posets $J$ essentially uses the Coxeter spectra of the simply laced Euclidean diagrams presented in Figure 1.

The nonsymmetric Gram matrix $\check{G}_{\Delta}$ of any graph $\Delta=$ $\left(\Delta_{0}, \Delta_{1}\right) \in\left\{\widetilde{\mathbb{D}}_{n}, n \geq 4, \widetilde{\mathbb{E}}_{6}, \widetilde{\mathbb{E}}_{7}, \widetilde{\mathbb{E}}_{8}\right\}$ of Figure 1 , with the set of vertices $\Delta_{0}=\{1, \ldots, n, n+1\}$ and the set of edges $\Delta_{1}$, is defined to be the matrix

$$
\check{G}_{\Delta}=\left[\begin{array}{ccccc}
1 & d_{12}^{\Delta} & \ldots & d_{1 n}^{\Delta} & d_{1 n+1}^{\Delta} \\
0 & 1 & \ldots & d_{2 n}^{\Delta} & d_{2 n+1}^{\Delta} \\
\vdots & \vdots & \ddots & \vdots & \vdots \\
0 & 0 & \ldots & 1 & d_{n n+1}^{\Delta} \\
0 & 0 & \ldots & 0 & 1
\end{array}\right] \in \mathbb{M}_{n+1}(\mathbb{Z}),
$$

where $d_{i j}^{\Delta}=-\left|\Delta_{1}(i, j)\right|$, if there is an edge $\bullet_{i}-\bullet_{j}$ and $i \leq j$. We set $d_{i j}^{\Delta}=0$, if $\Delta_{1}(i, j)$ is empty or $j<i$.

The Coxeter polynomial $\operatorname{cox}_{\Delta}(t):=\operatorname{det}\left(t \cdot E+\check{G}_{\Delta} \cdot \check{G}_{\Delta}^{-\operatorname{tr}}\right)$ of any diagram $\Delta=\left(\Delta_{0}, \Delta_{1}\right) \in\left\{\widetilde{\mathbb{D}}_{n}, n \geq 4, \widetilde{\mathbb{E}}_{6}, \widetilde{\mathbb{E}}_{7}, \widetilde{\mathbb{E}}_{8}\right\}$ does not depend on the numbering of the vertices in $\Delta_{0}$ and is presented in (48). If $n \geq 1$ and $\Delta=\widetilde{\mathbb{A}}_{n}$, the Coxeter polynomial $\operatorname{cox}_{\Delta}(t):=\operatorname{det}\left(t \cdot E+\check{G}_{\Delta} \cdot \check{G}_{\Delta}^{-\operatorname{tr}}\right)$ of $\Delta$ depends on the numbering of the vertices in $\Delta_{0}$ and is one of 


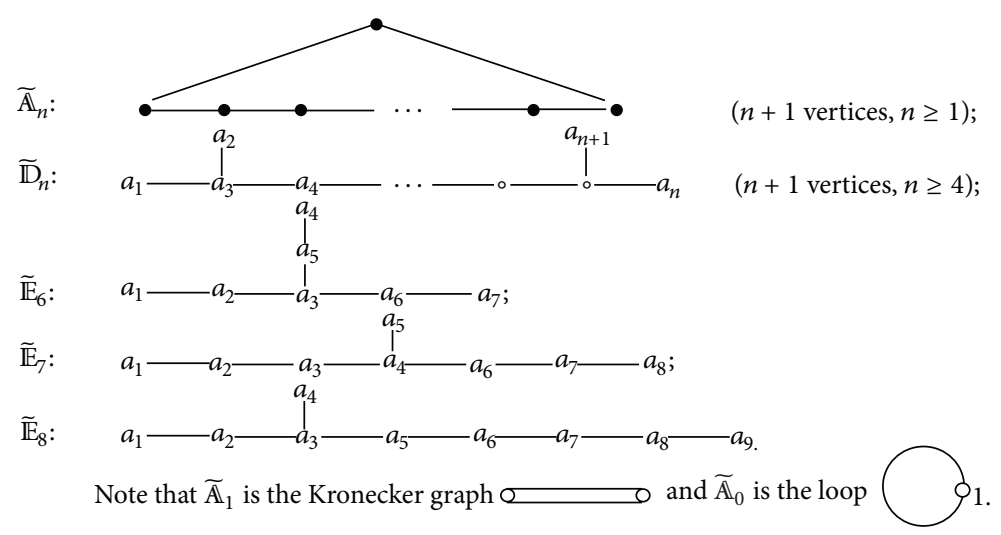

Figure 1: Simply laced Euclidean (extended Dynkin) diagrams.

the polynomials $F_{\Delta}^{(1)}(t), F_{\Delta}^{(2)}(t), \ldots, F_{\Delta}^{\left(m_{n}\right)}(t)$ presented in [4], where

$$
\begin{aligned}
& F_{\Delta}^{(j)}(t)=t^{n+1}-t^{n-j+1}-t^{j}+1 \\
&=(t-1)^{2} \cdot \mathfrak{v}_{j}(t) \cdot \mathfrak{b}_{n-j+1}(t), \\
& m_{n}= \begin{cases}\frac{n}{2}, & \text { if } n \text { is even, } \\
\frac{n+1}{2}, & \text { if } n+1 \text { is even, }\end{cases}
\end{aligned}
$$

for $j=1, \ldots, m_{n}$, and $\mathfrak{v}_{m}(t)=t^{m-1}+t^{m-2}+\cdots+t^{2}+t+1$. In particular, if $n+1$ is even and $j=m_{n}=(n+1) / 2$, then $t^{n-j+1}=t^{j}$ and

$$
F_{\Delta}^{\left(m_{n}\right)}(t)=F_{\Delta}^{((n+1) / 2)}(t)=t^{n+1}-2 t^{(n+1) / 2}+1 .
$$

Following [4, 21], we associate (in Section 2) to any principal poset $J$ a simply laced Euclidean diagram $D J \in$ $\left\{\widetilde{\mathbb{A}}_{n}, n \geq 1, \widetilde{\mathbb{D}}_{n}, n \geq 4, \widetilde{\mathbb{E}}_{6}, \widetilde{\mathbb{E}}_{7}, \widetilde{\mathbb{E}}_{8}\right\}$ such that the incidence symmetric Gram matrix $G_{J}:=(1 / 2)\left[C_{J}+C_{J}^{\mathrm{tr}}\right] \in \mathbb{M}_{J}(\mathbb{Q})$ is $\mathbb{Z}$-congruent to the symmetric Gram matrix

$$
G_{D J}:=\frac{1}{2}\left[\check{G}_{D J}+\check{G}_{D J}^{\operatorname{tr}}\right] \in \mathbb{M}_{D J}(\mathbb{Q})=\mathbb{M}_{J}(\mathbb{Q})
$$

of $D J$; that is, there is a $\mathbb{Z}$-invertible matrix $B$ such that $G_{D J}=$ $B^{\operatorname{tr}} \cdot G_{J} \cdot B$.

One of the aims of the Coxeter spectral analysis of nonnegative finite posets is to study the question when the Coxeter type

$$
\operatorname{Ctype}_{J}:=\left(\operatorname{specc}_{J}, \mathbf{c}_{J}, \check{\mathbf{c}}_{J}\right)
$$

of a poset $J$ determines the matrix $C_{J}$ (and, hence, the poset $J$ ) uniquely, up to a $\mathbb{Z}$-congruency. Here, we set $\check{\mathbf{c}}_{J}=\mathbf{c}_{J}$, if $J$ is positive. In other words, we claim that, for any pair $J, I$ of nonnegative one-peak posets, Ctype $\mathbf{e}_{J}=\mathbf{C t y p e}_{I}$ if and only if the incidence matrices $C_{J}$ and $C_{I}$ are $\mathbb{Z}$-congruent. We also study the problem related with the results proved by Horn and Sergeichuk [35], if for any $\mathbb{Z}$-invertible matrix $A \in \mathbb{M}_{n}(\mathbb{Z})$, there exists $B \in \mathbb{M}_{n}(\mathbb{Z})$ such that $A^{\operatorname{tr}}=B^{\operatorname{tr}} \cdot A \cdot B$ and $B^{2}=E$ is the identity matrix; see $[17,18]$.

The main results of the present paper on nonnegative posets $J$ can be summarised as follows:

(1) canonical equivalences between the incidences, Tits, and Euler quadratic form (and corresponding Coxeter transformations and Coxeter spectra) of any poset $J$, established in Proposition 5;

(2) a characterization of principal posets given in Section 3. We show that a connected poset $J$ is principal if and only if there exists a simply laced Euclidean diagram

$$
D J \in\left\{\widetilde{\mathbb{D}}_{n}, n \geq 4, \widetilde{\mathbb{E}}_{6}, \widetilde{\mathbb{E}}_{7}, \widetilde{\mathbb{E}}_{8}\right\}
$$

such that the symmetric Gram matrix $G_{J}:=(1 / 2)\left[C_{J}+C_{J}^{\operatorname{tr}}\right] \epsilon$ $\mathbb{M}_{J}(\mathbb{Q})$ of $J$ is $\mathbb{Z}$-congruent to the symmetric Gram matrix $G_{D J}:=(1 / 2)\left[\check{G}_{D J}+\check{G}_{D J}^{\mathrm{tr}}\right] \in \mathbb{M}_{D J}(\mathbb{Q})$ of $D J$. Moreover, we show in Section 3 that, given a connected principal poset $J$, the Coxeter spectrum specc $_{J}$ is a subset of a unit circle $\mathcal{S}^{1}=$ $\{z \in \mathbb{C} ;|z|=1\}, 1 \in \operatorname{specc}_{J}$, and any $z \in \operatorname{specc}_{J}$ is a root of unity;

(3) a Coxeter spectral classification result (Corollary 11) asserting that, given a pair $I, J$ of one-peak principal posets with at most 13 elements, the following conditions are equivalent:

(3a) $D I=D J$,

(3b) $\operatorname{specc}_{I}=$ specc $_{J}$,

(3c) $\check{\mathbf{c}}_{I}=\check{\mathbf{c}}_{J}$ and $|I|=|J|$,

(3d) the incidence matrix $C_{I} \in \mathbb{M}_{I}(\mathbb{Z})$ is $\mathbb{Z}$-congruent to the incidence matrix $C_{I} \in \mathbb{M}_{I}(\mathbb{Z})$; that is, there is a $\mathbb{Z}$-invertible matrix $B$ such that $C_{I}=B^{\operatorname{tr}} \cdot C_{J} \cdot B$.

In Section 3, we study principal posets by means of the defect and the reduced Coxeter number, and in Section 4, we present a framework for the study of nonnegative posets of corank $r \geq 2$ by means of their defect and the reduced Coxeter number. Examples are given in Sections 3-5.

The reader is referred to $[1,16,17,26]$ for a background of poset representation theory and elementary introduction to the poset matrix problems. 


\section{A Framework for the Coxeter Spectral Analysis of Finite Posets}

The quadratic wanderings on finite posets $J$ studied in [1] are playing a key role in the representation theory of posets, algebras, and coalgebras, as well as in the algebraic combinatorics of posets; see $[6,9-14,16,24-26,28,31,32$, 36-39]. Except for the incidence wandering and the Euler wanderings defined by the incidence matrix $C_{J} \in \mathbb{M}_{m}(\mathbb{Z}) \equiv$ $\mathbb{M}_{J}(\mathbb{Z})(2)$, with $\operatorname{det} C_{J}=1$ and a fixed numbering $J=$ $\left\{a_{1}, \ldots, a_{m}\right\}$, as well as the Euler matrix $\bar{C}_{J}:=C_{J}^{-1}$, we study in $[1,26-28]$ the Tits wandering defined by the Tits matrix $\widehat{C}_{J} \in$ $\mathbb{M}_{m}(\mathbb{Z}) \equiv \mathbb{M}_{J}(\mathbb{Z})$ of $J$ (see $\left.[28,(3.6)]\right)$, that is, the Gram matrix of the Tits $\mathbb{Z}$-bilinear form $\widehat{b}_{J}: \mathbb{Z}^{J} \times \mathbb{Z}^{J} \rightarrow \mathbb{Z}$ given by

$$
\begin{aligned}
\widehat{b}_{J}(x, y)= & \sum_{a_{i} \in J} x_{i} y_{i}+\sum_{a_{j}<a_{i} \in \check{J}} x_{i} y_{j} \\
& -\sum_{a_{p} \in \max J a_{i}<a_{p}} \sum_{i} y_{p}=x \cdot \widehat{C}_{J} \cdot y^{\mathrm{tr}},
\end{aligned}
$$

where $\max J$ is the set of all maximal elements in the poset $J$ and $\check{J}:=J \backslash \max J$. We call $\widehat{q}_{J}(x):=\widehat{b}_{J}(x, x)=x \cdot \widehat{C}_{J} \cdot x^{\text {tr }}$ the Tits quadratic form of $J$.

A homological interpretation of the $\mathbb{Z}$-bilinear forms $\widehat{b}_{J}(x, y)=x \cdot \widehat{C}_{J} \cdot y^{\operatorname{tr}}$ and $\bar{b}_{J}(x, y)=x \cdot \bar{C}_{J} \cdot y^{\operatorname{tr}}$ is given in $[1,(1.3)]$. For a geometric interpretation of the Tits form $\widehat{q}_{I}$ of a one-peak poset $I$, the reader is referred to Drozd [32] and Simson [26].

Note that, given a one-peak poset $I$ of the form $I=$ $\{1,2, \ldots, n, *=n+1\}$, with a unique maximal element $*=$ $n+1$, we have

$$
\widehat{C}_{I}=\left[\begin{array}{c|c}
C_{T}^{\mathrm{tr}} & -u \\
\hline 0 & 1
\end{array}\right] \in \mathbb{M}_{n+1}(\mathbb{Z}), \quad \text { with } u=\left[\begin{array}{c}
1 \\
\vdots \\
1
\end{array}\right],
$$

where $C_{T} \in \mathbb{M}_{T}(\mathbb{Z})=\mathbb{M}_{n}(\mathbb{Z})$ is the incidence matrix of the poset $T=I \backslash\{*\}=\{1,2, \ldots, n\}$; see [26]. Note that $\widehat{q}_{I}(x)=$ $x \cdot \widehat{C}_{I} \cdot x^{\text {tr }}$.

Now, we show that, in the Coxeter spectral study of finite posets $J$, we can use the Coxeter spectral technique introduced in $[2,4]$, for the edge-bipartite graphs (signed graphs [5]), and developed in [2, 34, 40] for the matrix morsifications of unit quadratic forms.

Following $[3,4,24]$, by an edge-bipartite graph (bigraph, in short), we mean a pair $\Delta=\left(\Delta_{0}, \Delta_{1}\right)$, where $\Delta_{0}$ is a finite nonempty set of vertices and $\Delta_{1}$ is a finite set of edges equipped with a bipartition $\Delta_{1}=\Delta_{1}^{-} \cup \Delta_{1}^{+}$such that the set $\Delta_{1}(i, j)=\Delta_{1}^{-}(i, j) \cup \Delta_{1}^{+}(i, j)$ of edges connecting the vertices $i$ and $j$ does not contain edges lying in $\Delta_{1}^{-}(i, j) \cap \Delta_{1}^{+}(i, j)$, for each pair of vertices $i, j \in \Delta_{0}$, and either $\Delta_{1}(i, j)=\Delta_{1}^{-}(i, j)$ or $\Delta_{1}(i, j)=\Delta_{1}^{+}(i, j)$. Note that the edge-bipartite graphs can be viewed as signed multigraphs satisfying a separation property; see $[4,5]$.

We visualize $\Delta$ as a graph in a Euclidean space $\mathbb{R}^{m}, m \geq 2$, with the vertices numbered by the integers $1, \ldots, n$; usually, we simply write $\Delta_{0}=\{1, \ldots, n\}$. An edge in $\Delta_{1}^{-}(i, j)$ is visualised as a continuous one $\bullet_{i}-\bullet_{j}$, and an edge in $\Delta_{1}^{+}(i, j)$ is visualised as a dotted one $\bullet_{i}--\bullet_{j}$. A bigraph $\Delta$ is said to be loop-free if it has no loops.

We view any finite graph $\Delta=\left(\Delta_{0}, \Delta_{1}\right)$ as an edgebipartite one by setting $\Delta_{1}^{-}(i, j)=\Delta_{1}(i, j)$ and $\Delta_{1}^{+}(i, j)=\emptyset$, for each pair of vertices $i, j \in \Delta_{0}$.

To any loop-free edge-bipartite graph $\Delta=\left(\Delta_{0}, \Delta_{1}\right)$, with a fixed numbering $\Delta_{0}=\left\{a_{1}, \ldots, a_{m}\right\}$ of its vertices, we associate the upper-triangular nonsymmetric Gram matrix $\check{G}_{\Delta}=$ $E+\left[d_{i j}^{\Delta}\right] \in \mathbb{M}_{m}(\mathbb{Z})$ of the form (20), with $m:=n+1$, where $d_{i j}^{\Delta}=-\left|\Delta_{1}^{-}(i, j)\right|$, if there is an edge $\bullet_{i}-\bullet_{j}$ and $i \leq j$, $d_{i j}^{\Delta}=\left|\Delta_{1}^{+}(i, j)\right|$, if there is an edge $\bullet_{i^{-}-\bullet_{j}}$ and $i \leq j$. We set $d_{i j}^{\Delta}=0$, if $\Delta_{1}(i, j)$ is empty or $j<i$. Since $\Delta$ is loop-free, we have $d_{11}^{\Delta}=\cdots=d_{m m}^{\Delta}=0$ and the main diagonal of $\check{G}_{\Delta}$ consists of unities.

Following [4], we call $\Delta=\left(\Delta_{0}, \Delta_{1}\right)$ positive (resp., nonnegative), if the symmetric Gram matrix

$$
G_{\Delta}:=(1 / 2)\left(\check{G}_{\Delta}+\check{G}_{\Delta}^{\mathrm{tr}}\right)
$$

of $\Delta$ is positive definite (resp., positive semidefinite).

Following [4], we associate to any loop-free edge-bipartite graph $\Delta$, with $\left|\Delta_{0}\right|=n \geq 2$, the Coxeter spectrum specc $_{\Delta} \subseteq \mathbb{C}$ defined to be the spectrum of the Coxeter (-Gram) matrix

$$
\operatorname{Cox}_{\Delta}:=-\check{G}_{\Delta} \cdot \check{G}_{\Delta}^{-\operatorname{tr}} \in \mathbb{M}_{n}(\mathbb{Z}),
$$

the Coxeter polynomial

$$
\operatorname{cox}_{\Delta}(t):=\operatorname{det}\left(t \cdot E-\operatorname{Cox}_{\Delta}\right) \in \mathbb{Z}[t],
$$

the Coxeter transformation $\Phi_{\Delta}: \mathbb{Z}^{n} \rightarrow \mathbb{Z}^{n}$, given by $x \mapsto$ $\Phi_{\Delta}(x):=x \cdot \operatorname{Cox}_{\Delta}$, the Coxeter number $\mathbf{c}_{\Delta}$ (the order of $\Phi_{\Delta}$ in the automorphism group of $\mathbb{Z}^{n}$, i.e., the minimal integer $r \geq 1$ such that $\left.\Phi_{\Delta}^{r}=E\right)$, the $\mathbb{Z}$-bilinear Gram form $b_{\Delta}$ : $\mathbb{Z}^{n} \times \mathbb{Z}^{n} \rightarrow \mathbb{Z}$ of $\Delta$ given by $b_{\Delta}(x, y):=x \cdot \check{G}_{\Delta} \cdot y^{\operatorname{tr}}$, and the integral unit quadratic form

$$
\begin{aligned}
q_{\Delta}(x):= & b_{\Delta}(x, x)=x_{1}^{2}+\cdots+x_{n}^{2} \\
& +\sum_{i<j} d_{i j}^{\Delta} x_{i} x_{j}=x \cdot G_{\Delta} \cdot x^{\mathrm{tr}} .
\end{aligned}
$$

Conversely, following Ovsienko [24], to any integral unit form

$$
q(x)=x_{1}^{2}+\cdots+x_{n}^{2}+\sum_{i<j} q_{i j} x_{i} x_{j}, \quad \text { with } q_{i j} \in \mathbb{Z},
$$

we associate the loop-free bigraph bigr ( $q$ ) of $q$ as follows (see also [34, 41]):

(a) the vertices of $\operatorname{bigr}(q)$ are the integers $1, \ldots, n$,

(b) two vertices $i \neq j$ are joined by $-q_{i j}$ continuous edges of the form $\bullet_{i}-\bullet_{j}$ if $q_{i j}$ is negative, and by $q_{i j}$ dotted edges of the form $\bullet^{---\bullet_{j}}$, if $q_{i j}$ is positive,

(c) there is no edge between $i$ and $j$, if $q_{i j}=0$, or $i=j$. 
To any poset $J \equiv(J, \preceq)$, with a fixed numbering $J=$ $\left\{a_{1}, \ldots, a_{m}\right\}$ of its points, we associate the following three edge-bipartite graphs:

$$
\Delta_{J}:=\operatorname{bigr}\left(q_{J}\right), \quad \widehat{\Delta}_{J}:=\operatorname{bigr}\left(\widehat{q}_{J}\right), \quad \bar{\Delta}_{J}:=\operatorname{bigr}\left(\bar{q}_{J}\right),
$$

where $\operatorname{bigr}\left(q_{J}\right)$, $\operatorname{bigr}\left(\hat{q}_{J}\right)$, and $\operatorname{bigr}\left(\bar{q}_{J}\right)$ are the bigraphs of the quadratic forms $q_{J}, \widehat{q}_{J}$, and $\bar{q}_{J}$, respectively; see (7). More precisely, the bigraphs (33) are defined as follows.

(i) The set of vertices of each of the bigraphs $\Delta_{J}, \widehat{\Delta}_{J}$, and $\bar{\Delta}_{J}$ is the enumerated set $J=\left\{a_{1}, \ldots, a_{m}\right\}$.

(ii) There is an edge $a_{i^{--}-a_{j}}$ in $\Delta_{J}$, if $a_{i} \prec a_{j}$ or $a_{j} \prec a_{i}$ holds in $J$.

(iii) There is an edge $a_{i---a_{j}}$ in $\widehat{\Delta}_{J}$, if $a_{i}$ and $a_{j}$ are not maximal in $J$ and $a_{i}<a_{j}$ or $a_{j}<a_{i}$ holds in $J$. There is an edge $a_{i}-a_{j}$ in $\widehat{\Delta}_{J}$, if $a_{i}<a_{j}$ holds and $a_{j}$ is maximal in $J$.

(iv) Let $\bar{C}_{J}=C_{J}^{-1}=\left[\bar{c}_{i j}\right] \in \mathbb{M}_{m}(\mathbb{Z})$ be the Euler matrix of $J$. There is an edge $a_{i^{-}}-a_{j}$ (resp., $a_{i}-a_{j}$ ) in $\bar{\Delta}_{J}$, if $\bar{c}_{i j}>0$ or $\bar{c}_{j i}>0$ (resp., $\bar{c}_{i j}<0$ or $\left.\bar{c}_{j i}<0\right)$.

We call $\Delta_{J}, \widehat{\Delta}_{J}$, and $\bar{\Delta}_{J}$ the incidence bigraph of $\Delta$, the Tits bigraph of $\Delta$, and the Euler bigraph of $\Delta$, respectively, (with respect to the numbering $J=\left\{a_{1}, \ldots, a_{m}\right\}$ ).

The following simple lemma is of importance.

Lemma 3. Assume that $J$ is a finite poset with a fixed numbering $J=\left\{a_{1}, \ldots, a_{m}\right\}$, and let $\Delta_{I}, \widehat{\Delta}_{I}, \bar{\Delta}_{I}$ be the loopfree edge-bipartite graphs associated with $J$ in (33).

(a) The symmetric Gram matrices $G_{J}, \widehat{G}_{J}, \bar{G}_{J}$ are $\mathbb{Z}$ congruent to the symmetric Gram matrices $G_{\Delta_{J}}, G_{\widehat{\Delta}_{J}}$, $G_{\bar{\Delta}_{I}}$, respectively. The rank of each of the symmetric Gram matrices $G_{\Delta_{I}}, G_{\widehat{\Delta}_{I}}, G_{\bar{\Delta}_{I}}$ does not depend of the numbering $J=\left\{a_{1}, \ldots, a_{m}\right\}$ and coincides with the common rank $\mathrm{rk} G_{\Delta_{J}}=\operatorname{rk} G_{\widehat{\Delta}_{J}}=\operatorname{rk} G_{\bar{\Delta}_{J}}$.

(b) $P_{J}(t)=P_{\Delta_{J}}(t)=P_{\widehat{\Delta}_{J}}(t)$.

(c) The poset $J$ is positive (resp., nonnegative) if and only if the bigraph $\Delta_{J}$ (and $\widehat{\Delta}_{J}, \bar{\Delta}_{J}$ ) is positive (resp., nonnegative).

(d) The poset $J$ is principal if and only if the bigraph $\Delta_{J}\left(\right.$ and $\left.\widehat{\Delta}_{J}, \bar{\Delta}_{J}\right)$ is principal.

Proof. For the proof of (a), we recall that the Gram matrices $G_{J}, \widehat{G}_{J}, \bar{G}_{J}, G_{\Delta_{J}}, G_{\widehat{\Delta}_{J}}, G_{\bar{\Delta}_{J}}$ are invariant, up to $\mathbb{Z}$-congruency, under permutations of the elements $\left\{a_{1}, \ldots, a_{m}\right\}$. Since $J$ admits an upper-triangular numbering $J^{\prime}=\left\{a_{1}^{\prime}, \ldots, a_{m}^{\prime}\right\}$ and $G_{\Delta_{I^{\prime}}}=G_{J^{\prime}}$, then (a) follows. The proof of remaining statements is left to the reader.

Following the terminology used in $[2-4,34]$, we introduce the following definition.
Definition 4. Let $J$ be a finite poset, with a fixed numbering $J=\left\{a_{1}, \ldots, a_{m}\right\}$.

(a) We associate with $J$ the following three Coxeter matrices:

(al) the (incidence) Coxeter matrix $\operatorname{Cox}_{J}:=-C_{J}$. $C_{J}^{-\operatorname{tr}} \in \mathbb{M}_{m}(\mathbb{Z})$

(a2) the Coxeter-Tits matrix $\widehat{\mathrm{Cox}}_{J}:=-\widehat{C}_{J} \cdot \widehat{C}_{J}^{-\operatorname{tr}} \in$ $\mathbb{M}_{m}(\mathbb{Z})$

(a3) the Coxeter-Euler matrix $\overline{\operatorname{Cox}}_{J}:=-C_{J}^{-1} \cdot C_{J}^{\text {tr }} \epsilon$ $\mathbb{M}_{m}(\mathbb{Z})$

Moreover, we define the following three Coxeter transformations:

(a4) the (incidence) Coxeter transformation $\Phi_{m}$ : $\mathbb{Z}^{m} \rightarrow \mathbb{Z}^{m}$ of $J$

(a5) the Coxeter-Tits transformation $\widehat{\Phi}_{J}: \mathbb{Z}^{m} \rightarrow$ $\mathbb{Z}^{m}$ of $J$

(a6) the Coxeter-Euler transformation $\bar{\Phi}_{J}: \mathbb{Z}^{m} \rightarrow$ $\mathbb{Z}^{m}$ of $J$, by the following formulae:

$$
\begin{aligned}
& \Phi_{J}(x)=x \cdot \operatorname{Cox}_{J}, \quad \widehat{\Phi}_{J}(x)=x \cdot \widehat{\operatorname{Cox}}_{J}, \\
& \text { and } \bar{\Phi}_{J}(x)=x \cdot \overline{\operatorname{Cox}}_{J} .
\end{aligned}
$$

(b) The integral polynomial

$$
\begin{aligned}
\operatorname{cox}_{J}(t) & =\operatorname{det}\left(t \cdot E-\operatorname{Cox}_{J}\right)=\operatorname{det}\left(t \cdot E-\widehat{\operatorname{Cox}}_{J}\right) \\
& =\operatorname{det}\left(t \cdot E-\overline{\operatorname{Cox}}_{J}\right) \in \mathbb{Z}[t]
\end{aligned}
$$

is called the Coxeter polynomial of the poset $J$.

(c) The Coxeter spectrum of $J$ is the set specc $_{J} \subseteq \mathbb{C}$ of all $m=|J|$ eigenvalues of the matrix $\operatorname{Cox}_{J}$, or, equivalently, the set $\operatorname{specc}_{J}$ of all $m=|J|$ roots of the Coxeter polynomial $\operatorname{cox}_{J}(t)$.

(d) The order $\mathbf{c}_{J}:=\operatorname{ord}\left(\Phi_{J}\right)$ of the Coxeter transformation $\Phi_{J}: \mathbb{Z}^{J} \rightarrow \mathbb{Z}^{J}$ is called the Coxeter number of the poset $J$. In other words, $\mathbf{c}_{J}$ is the minimal integer $r \geq 1$ such that $\Phi_{J}^{r}=i d$. We set $\mathbf{c}_{J}=\infty$, if $\Phi_{J}^{r} \neq i d$, for any $r \geq 1$.

(e) Assume that $J$ is nonnegative. The Coxeter type of $J$ is defined to be the pair Ctype $_{J}:=\left(\operatorname{specc}_{J}, \mathbf{c}_{J}\right)$ if $J$ is positive, and the triple $\operatorname{Ctype}_{J}:=\left(\operatorname{specc}_{J}, \mathbf{c}_{J}, \check{\mathbf{c}}_{J}\right)$ if $J$ is not positive, where $\check{\mathbf{c}}_{J}$ is the reduced Coxeter number of $J$ in the sense of Theorems 10 and 18.

The following proposition shows that equality (35) holds.

Proposition 5. Let $J$ be a finite poset, with a fixed numbering $J=\left\{a_{1}, \ldots, a_{m}\right\}$, let $q_{J}, \widehat{q}_{J}, \bar{q}_{J}: \mathbb{Z}^{m} \rightarrow \mathbb{Z}$ be the incidence, Tits, and Euler quadratic form of $J$, and let $\Phi_{J}, \widehat{\Phi}_{J}, \bar{\Phi}_{J}: \mathbb{Z}^{m} \rightarrow \mathbb{Z}^{m}$ be the corresponding Coxeter transformations. 
(a) The following equalities hold $\widehat{C}_{J}=B \cdot \bar{C}_{J} \cdot B^{t r}$ and $C_{J}^{t r}=B^{\prime} \cdot \bar{C}_{J} \cdot B^{\prime t r}$, and the following diagrams are commutative
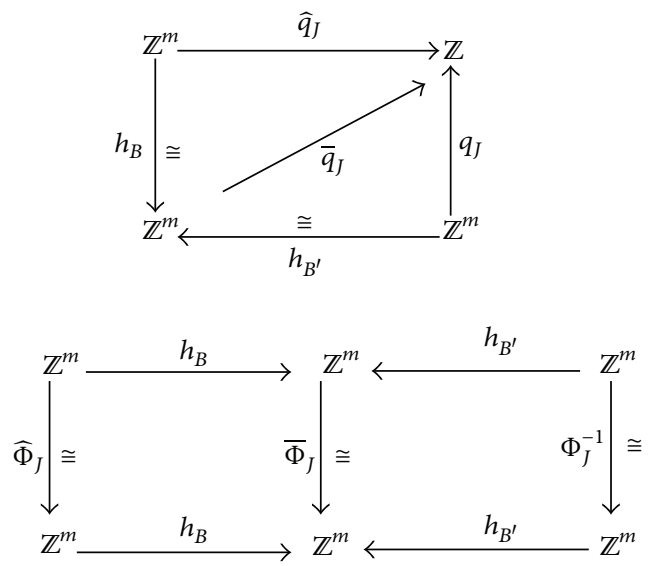

where $B^{\prime}=C_{J}^{t r}, B=\left[\begin{array}{c|c}C_{\check{J}} & 0 \\ \hline 0 & E\end{array}\right], \check{J}=J \backslash \max J$, and $h_{B}, h_{B^{\prime}}: \mathbb{Z}^{m} \rightarrow \mathbb{Z}^{m}$ are the group isomorphisms defined by the formulae $h_{B}(x)=x \cdot B$ and $h_{B^{\prime}}(x)=$ $x \cdot B^{\prime}$, for $x \in \mathbb{Z}^{m}$.

(b) $\widehat{C}_{o x_{J}}=B \cdot \bar{C} o x_{J} \cdot B^{-1}, \operatorname{Cox}_{J}^{-1}=\operatorname{Cox}_{J^{o p}}=B^{\prime} \cdot \bar{C} o x_{J}$. $B^{\prime-1}$, and $\Phi_{J^{o p}}=\Phi_{J}^{-1}$.

(c) The Coxeter number $\mathbf{c}_{J}=\operatorname{ord}\left(\Phi_{J}\right)$ of the poset $J$ coincides with the Coxeter number of $J^{o p}$. Moreover, $\mathbf{c}_{J}=\operatorname{ord}\left(\widehat{\Phi}_{J}\right)=\operatorname{ord}\left(\bar{\Phi}_{J}\right)$ and $\operatorname{cox}_{J^{o p}}(t)=\operatorname{cox} x_{J}(t)$.

(d) Assume that $J$ is connected and nonnegative.

(d1) If the numbering $J=\left\{a_{1}, \ldots, a_{m}\right\}$ is uppertriangular and $\Delta_{J}$ is the bigraph (33) associated to $J$, then $\operatorname{Cox}_{\Delta_{J}}=\operatorname{Cox}_{J}$ and $\operatorname{cox}{\Delta_{J}}(t)=\operatorname{cox}_{J}(t)$.

(d2) The Coxeter type Ctype $_{I}:=\left(\operatorname{specc}_{J}, \mathbf{c}_{J}, \check{\mathbf{c}}_{J}\right)$ of $J$ does not depend on the numbering $J=$ $\left\{a_{1}, \ldots, a_{m}\right\}$.

(d3) The Coxeter spectrum specc $_{J}$ is a subset of a unit circle $\mathcal{S}^{1}=\{z \in \mathbb{C} ;|z|=1\}$, and any $z \in \operatorname{specc}_{J}$ is a root of unity.

(d4) The poset $J$ is positive if and only if $1 \notin \operatorname{specc}_{J}$.

Proof. The first equality $\widehat{C}_{J}=B \cdot \bar{C}_{J} \cdot B^{\text {tr }}$ is obvious, and the second one $C_{I}^{\mathrm{tr}}=B^{\prime} \cdot \bar{C}_{J} \cdot B^{\text {tr }}$ follows by a direct calculation. Hence, (b) follows and, consequently, the diagrams (36) are commutative; see [1, Proposition 3.13]. Hence, the statement (c) follows from the commutativity of the diagrams (36).

(d1) We recall from Section 1 that, given two numberings $J=\left\{a_{1}, \ldots, a_{m}\right\}$ and $J^{\prime}=\left\{a_{1}^{\prime}, \ldots, a_{m}^{\prime}\right\}$ of elements in $J$, we have $C_{J^{\prime}}=\widehat{\sigma}^{-1} \cdot C_{J} \cdot \widehat{\sigma}$, where $\widehat{\sigma} \in \operatorname{Gl}(m, \mathbb{Z})$ is the permutation matrix of a permutation $\sigma \in \mathbf{S}_{m}$. Hence, (d1) easily follows. (d2) It is sufficient to note that the incidence matrix $C_{J}$ is upper triangular. Hence, $C_{J}=\check{G}_{\Delta_{I}}$ and $\operatorname{Cox}_{\Delta_{I}}=$ $\mathrm{Cox}_{J}$.

To prove (d3) and (d4), we recall from [2] and [3, Proposition 2.6] that the Coxeter spectrum specc $_{A}$ of any matrix morsification $A \in$ Mor $_{\Delta}$ of a nonnegative bigraph $\Delta$ is a subset of the unit circle $\delta^{1}$ and any $z \in \operatorname{specc}_{A}$ is a root of unity (see also $[41,42]$ ). Moreover, $\Delta$ is positive iff $1 \notin \operatorname{specc}_{A}$. Assume that $J$ is connected and nonnegative. Then, the bigraph $\Delta_{J}(33)$ associated to $J$ is nonnegative, $A$ := $\check{G}_{\Delta_{J}}=\nabla\left(C_{J}\right)$ is a morsification of $\Delta_{J}$, and specc $_{J}=$ specc $_{A}$, because the incidence matrix $C_{J}$ is quasitriangular and [4, Proposition 2.2] applies. This completes the proof.

Corollary 6. For any poset J, equality (35) holds.

Proof. Apply Proposition 5(b).

The following example shows that the correspondence $J \mapsto \Delta_{J}$ defined in (33) does not preserve the Coxeter types of $J$ and $\Delta_{J}$. In particular, it shows that the equality $\operatorname{cox}_{J}(t)=$ $\operatorname{cox}_{\Delta_{I}}(t)$ does not hold in general and the Coxeter polynomial $\operatorname{cox}_{\Delta_{I}}(t)$ depends on the numbering of $J$, whereas the Coxeter polynomial $\operatorname{cox}_{J}(t)$ does not depend on the numbering of $J$.

Example 7. Consider the poset $J$ such that its Hasse quiver has the form

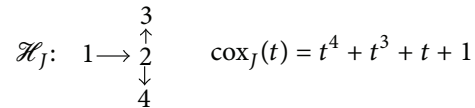

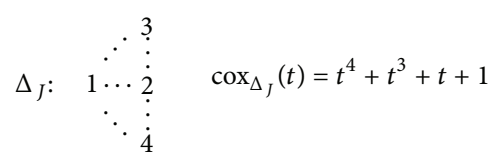

By a permutation of the elements in $J$, we get

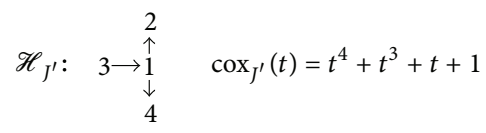

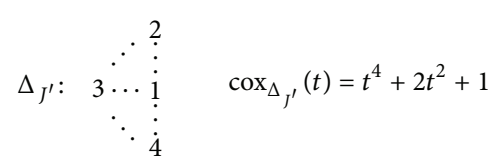

Note that the first numbering is upper-triangular, whereas the second one is not upper-triangular.

\section{Principal Posets}

We recall that a poset $J$ is principal if its incidence unit form $q_{J}$ is principal in the sense of [34, Definition 2.1]; that is, $q_{J}: \mathbb{Z}^{J} \rightarrow \mathbb{Z}$ is nonnegative and not positive, and the kernel $\operatorname{Ker} q_{J}:=\left\{v \in \mathbb{Z}^{J} ; q_{J}(v)=0\right\}$ is an infinite cyclic subgroup of $\mathbb{Z}^{J}$.

We start with the following useful observation. 
Lemma 8. Assume that $J$ is a connected principal poset.

(a) The Coxeter number $\mathbf{c}_{J}$ of $J$ is infinite.

(b) The Coxeter spectrum specc $_{J}$ is a subset of a unit circle $\mathcal{S}^{1}=\{z \in \mathbb{C} ;|z|=1\}, 1 \in \operatorname{specc}_{J}$, and any $z \in \operatorname{specc}_{J}$ is a root of unity.

(c) If $\operatorname{Ker} q_{J}=\mathbb{Z} \cdot \mathbf{h}$, then $\operatorname{Ker} \widehat{q}_{J}=\mathbb{Z} \cdot \widehat{\mathbf{h}}$ and $\operatorname{Ker} \bar{q}_{J}=$ $\mathbb{Z} \cdot \overline{\mathbf{h}}$, where

(i) $\overline{\mathbf{h}}=\mathbf{h} \cdot B^{\prime}, \overline{\mathbf{h}}=\widehat{\mathbf{h}} \cdot B, \widehat{\mathbf{h}}=\mathbf{h} \cdot B^{\prime} \cdot B^{-1}$,

(ii) $B^{\prime}=C_{J}^{t r}, B=\left[\begin{array}{c|c}C_{\check{J}} & 0 \\ \hline 0 & E\end{array}\right]$, and $\check{J}=J \backslash \max J$

are as in Proposition 5.

Proof. (a) By Proposition 5 (d2), $\mathbf{c}_{J}$ is independent of the numbering of $J$. Then, without loss of generality, we may suppose that the numbering of $J$ is upper-triangular. Then, by Lemma 3(d) and Proposition 5(d1), the Coxeter number $\mathbf{c}_{J}$ coincides with the Coxeter number of the principal edgebipartite graph $\Delta_{J}$ associated with $J$ in (33). Then, (a) is a consequence of [3, Proposition 3.12].

The statements (b) and (c) follow by applying Proposition 5 and the commutative diagram (36).

Proposition 9. Let $J$ be a connected poset, $m=|J| \geq 2$, and let $G_{J}, \widehat{G}_{J}, \bar{G}_{J}, \in \mathbb{M}_{J}(\mathbb{Q})$ be the symmetric incidence Gram matrix of $J$, the symmetric Tits-Gram matrix of $J$, and the symmetric Euler-Gram matrix of J, respectively. The following five conditions are equivalent.

(a) The poset $J$ is principal.

(b) The Gram matrix $G_{J}$ is positive indefinite of rank $m-1$.

(c) The Tits quadratic form $\hat{q}_{J}$ of $J$ is nonnegative and $\operatorname{Ker} \widehat{q}_{J}=\mathbb{Z} \cdot \widehat{\mathbf{h}}$, for some nonzero vector $\widehat{\mathbf{h}} \in \mathbb{Z}^{J}$.

(d) The Euler quadratic form $\bar{q}_{J}$ of $J$ is nonnegative and $\operatorname{Ker} \bar{q}_{J}=\mathbb{Z} \cdot \overline{\mathbf{h}}$, for some nonzero vector $\overline{\mathbf{h}} \in \mathbb{Z}^{J}$.

(e) If $G$ is any of the symmetric Gram matrices $G_{J}, \widehat{G}_{J}, \bar{G}_{J}, \in \mathbb{M}_{J}(\mathbb{Q})$ of $J$, then there exists a simply laced Euclidean diagram $D J \in\left\{\widetilde{\mathbb{A}}_{s}, s \geq 3, \widetilde{\mathbb{D}}_{n}, n \geq\right.$ $\left.4, \widetilde{\mathbb{E}}_{6}, \widetilde{\mathbb{E}}_{7}, \widetilde{\mathbb{E}}_{8}\right\}$ (uniquely determined by $J$ ) such that the matrix $G$ is $\mathbb{Z}$-congruent to the symmetric Gram matrix $G_{D J} \in \mathbb{M}_{D J}(\mathbb{Q})$ of the Euclidean diagram $D J$; that is, there is a $\mathbb{Z}$-invertible matrix $B \in G l(m, \mathbb{Z})$ such that $G_{D J}=B^{t r} \cdot G \cdot B$.

Proof. (a) $\Leftrightarrow$ (b) If $m=|J|$ and

$$
\begin{gathered}
D q_{J}: \mathbb{Z}^{m} \longrightarrow \mathbb{Z}^{m} \\
v \longmapsto D q_{J}(v)=\left(\frac{\partial q_{J}}{\partial x_{1}}(v), \ldots, \frac{\partial q_{J}}{\partial x_{m}}(v)\right),
\end{gathered}
$$

is the gradient group homomorphism of $q_{J}$, then $\operatorname{Ker} q_{J}=$ $\operatorname{Ker}\left[D q_{J}: \mathbb{Z}^{m} \rightarrow \mathbb{Z}^{m}\right]$ and the subgroup Ker $q_{J}$ of $\mathbb{Z}^{m}$ is of rank $m-\operatorname{rk} G_{J}$ and consists of all integral solutions of the system $2 \cdot G_{J} \cdot x^{\text {tr }}=0$ of linear equations with integral coefficients; see [34, Proposition 2.8]. Then, $(\mathrm{a}) \Leftrightarrow(\mathrm{b})$ follows.

The equivalences $(\mathrm{a}) \Leftrightarrow(\mathrm{c}) \Leftrightarrow(\mathrm{d})$ follow from Proposition 5 (a) and the commutativity of the diagram (36).

(e) $\Rightarrow$ (a) Assume that there exist a simply laced Euclidean diagram $D J \in\left\{\widetilde{\mathbb{A}}_{s}, s \geq 3, \widetilde{\mathbb{D}}_{n}, n \geq 4, \widetilde{\mathbb{E}}_{6}, \widetilde{\mathbb{E}}_{7}, \widetilde{\mathbb{E}}_{8}\right\}$ and a $\mathbb{Z}$ invertible matrix $B \in \mathrm{Gl}(m, \mathbb{Z})$ such that $G_{D J}=B^{\operatorname{tr}} \cdot G \cdot B$. It follows that the quadratic form $q_{D J}(x)=x \cdot G_{D J} \cdot x^{\text {tr }}$ is $\mathbb{Z}$ congruent to $q_{J}$ and $q_{J}=q_{D J} \circ h_{B}$. Then, (a) is a consequence of [36, Lemma VII.4.2].

(a) $\Rightarrow$ (e) Let $\bar{\Delta}_{J}$ be the Euler edge-bipartite graph defined in (33) of $J$. By (a) and Lemma 3 (d), $\bar{\Delta}_{J}$ is principal and the inflation algorithm defined in $[4,21]$ applies to $\Delta_{J}$. Consequently, there exists a simply laced Euclidean diagram $D J \in\left\{\widetilde{\mathbb{A}}_{s}, s \geq 3, \widetilde{\mathbb{D}}_{n}, n \geq 4, \widetilde{\mathbb{E}}_{6}, \widetilde{\mathbb{E}}_{7}, \widetilde{\mathbb{E}}_{8}\right\}$ and a $\mathbb{Z}$-invertible matrix $B \in \operatorname{Gl}(n, \mathbb{Z})$ defining the congruence $\bar{\Delta}_{J} \approx_{\mathbb{Z}} D J$; that is, the equality $G_{D J}=B^{\operatorname{tr}} \cdot G_{\bar{\Delta}_{J}} \cdot B=B^{\operatorname{tr}} \cdot G_{J} \cdot B$ holds. Then, in view of Proposition 5 , the implication $(\mathrm{a}) \Rightarrow(\mathrm{e})$ follows from Lemma 3 (d); see also Section 6.

Theorem 10. Let $J$ be a finite principal poset, with a numbering $\left\{a_{1}, \ldots, a_{m}\right\}$ of elements of J. Fix a nonzero vector $\mathbf{h}_{J} \in \mathbb{Z}^{J} \equiv$ $\mathbb{Z}^{m}$ such that $\operatorname{Ker} q_{J}=\mathbb{Z} \cdot \mathbf{h}_{J}$.

(a) There exist a minimal integer $\check{\mathbf{c}}_{J} \geq 2$ (called the reduced Coxeter number of $J$ ) and a group homomorphism $\partial_{J}$ : $\mathbb{Z}^{J} \rightarrow \mathbb{Z}$ (called the incidence defect of J) such that

$$
\begin{gathered}
\Phi_{J}^{\check{\mathbf{c}}_{J}}(v)=v+\partial_{J}(v) \cdot \mathbf{h}_{J}, \\
\partial_{J}\left(\Phi_{J}(v)\right)=\partial_{J}(v), \quad \forall v \in \mathbb{Z}^{J}, \\
\partial_{J}\left(\mathbf{h}_{J}\right)=0 .
\end{gathered}
$$

(b) Assume that $\check{\mathbf{c}}_{J} \geq 1$ and $\partial_{J}: \mathbb{Z}^{J} \rightarrow \mathbb{Z}$ are as in (a), and one sets $\overline{\mathbf{h}}_{J}=\mathbf{h}_{J} \cdot B^{\prime}, \widehat{\mathbf{h}}_{J}=\mathbf{h}_{J} \cdot B^{\prime} \cdot B^{-1}$, where $B^{\prime}, B \in \mathbb{M}_{J}(\mathbb{Z})$ are as in Proposition 5.

(b1) There exists a group homomorphism $\bar{\partial}_{J}: \mathbb{Z}^{J} \rightarrow$ $\mathbb{Z}$ (called the Euler defect of $J$ ) such that

$$
\begin{gathered}
\bar{\Phi}_{J}^{\check{c}_{J}}(v)=v+\bar{\partial}_{J}(v) \cdot \overline{\mathbf{h}}_{J}, \quad \forall v \in \mathbb{Z}^{J}, \\
\bar{\partial}_{J} \circ \Phi_{J}=\bar{\partial}_{J}, \\
\bar{\partial}_{J} \circ h_{B^{\prime}}=\partial_{J}, \quad \bar{\partial}_{J}\left(\overline{\mathbf{h}}_{J}\right)=0 .
\end{gathered}
$$

(b2) There exists a group homomorphism $\widehat{\partial}_{J}: \mathbb{Z}^{J} \rightarrow$ $\mathbb{Z}$ (called the Tits defect of $J$ ) such that

$$
\begin{gathered}
\widehat{\Phi}_{J}^{\check{c}_{J}}(v)=v+\widehat{\partial}_{J}(v) \cdot \widehat{\mathbf{h}}_{J}, \quad \forall v \in \mathbb{Z}^{J}, \\
\widehat{\partial}_{J}=\bar{\partial}_{J} \circ h_{B}=\partial_{J} \circ h_{B^{\prime}}^{-1} \circ h_{B}, \\
\widehat{\partial}_{J}=\widehat{\partial}_{J} \circ \Phi_{J}, \quad \widehat{\partial}_{J}\left(\widehat{\mathbf{h}}_{J}\right)=0 .
\end{gathered}
$$


(c) The Coxeter number $\mathbf{c}_{J}$ of $J$ is infinite, and the incidence defect $\partial_{J}: \mathbb{Z}^{J} \rightarrow \mathbb{Z}$ is nonzero.

(d) Given $v \in \mathbb{Z}^{J}$, the order $\mathbf{s}_{v}:=|\mathcal{O}(v)|$ of the $\Phi_{J}$-orbit $\mathcal{O}(v)$ is finite if and only if $\partial_{J}(v)=0$. If $\mathbf{s}_{v}=|\mathcal{O}(v)|$ is finite, then $\mathbf{s}_{v}$ divides $\check{\mathbf{c}}_{J}$ and there is a unique integer $m_{v}$ such that

$$
\begin{aligned}
m_{v} \cdot \mathbf{h} & =v+\Phi_{J}(v)+\Phi_{J}^{2}(v)+\cdots+\Phi_{J}^{\mathbf{s}_{v}-1}(v) \\
& =v+\Phi_{J}^{-1}(v)+\Phi_{J}^{-2}(v)+\cdots+\Phi_{J}^{-\mathbf{s}_{v}+1}(v) .
\end{aligned}
$$

Proof. We recall from the proof of Proposition 9 that

$$
\mathbb{Z} \cdot \mathbf{h}_{J}=\operatorname{Ker} q_{J}=\operatorname{Ker}\left[D q_{J}: \mathbb{Z}^{m} \longrightarrow \mathbb{Z}^{m}\right],
$$

where $m=|J| \geq 2$ and $D q_{J}: \mathbb{Z}^{m} \rightarrow \mathbb{Z}^{m}, v \mapsto$ $D q_{J}(v)=\left(\left(\partial q_{J} / \partial x_{1}\right)(v), \ldots,\left(\partial q_{J} / \partial x_{m}\right)(v)\right)$, is the gradient group homomorphism. It follows that $\mathbb{Z}^{m} / \mathbb{Z} \cdot \mathbf{h}_{J} \cong \operatorname{Im} D q_{J} \cong$ $\mathbb{Z}^{m-1}$. Denote by $\phi: \mathbb{Z}^{m} \rightarrow \mathbb{Z}^{m-1}$ the composite quotient epimorphism. Then, the form $q_{J}$ induces the form $\tilde{q}_{J}$ : $\mathbb{Z}^{m-1} \rightarrow \mathbb{Z}$ such that $\widetilde{q}_{J}(\phi(x))=q_{J}(x)$, for all $x \in \mathbb{Z}^{m}$. Moreover, the Coxeter transformation $\Phi_{J}: \mathbb{Z}^{m} \rightarrow \mathbb{Z}^{m}$ induces a group automorphism $\widetilde{\Phi}_{J}: \mathbb{Z}^{m-1} \rightarrow \mathbb{Z}^{m-1}$ such that

$$
\widetilde{\Phi}_{J} \circ \phi=\phi \circ \Phi_{J}, \quad \widetilde{q}_{J}\left(\widetilde{\Phi}_{J}(y)\right)=\widetilde{q}_{J}(y), \quad \forall y \in \mathbb{Z}^{m-1} .
$$

It follows that $\widetilde{q}_{J}$ is positive definite and there exists a minimal integer $\check{\mathbf{c}}_{J} \geq 1$ such that $\widetilde{\Phi}_{J}^{\check{\mathbf{c}}_{J}}$ is the identity map on $\mathbb{Z}^{m-1}$. Hence, (a) follows, because the equalities $\partial_{J}\left(\mathbf{h}_{J}\right)=0$ and $\partial_{J}\left(\Phi_{J}(v)\right)=\partial_{J}(v)$, for all $v \in \mathbb{Z}^{J}$, are almost obvious; see [34, Theorem 4.7].

In view of Proposition 5, the statements (b)-(d) are a consequence of (a) and Lemma 8(a). The reader is referred to [34, Theorem 4.7, Corollary 4.15] for more details.

Corollary 11. (a) If $J$ is a principal connected poset with at most 13 elements, then its Coxeter spectrum specc $_{J}$ is a subset of a unit circle $\mathcal{S}^{1}=\{z \in \mathbb{C} ;|z|=1\}, 1 \in$ specc $_{\text {J }}$, and any $z \in$ specc $_{J}$ is an mth root of unity, where $m \leq \check{\mathbf{c}}_{J}$ and $\check{\mathbf{c}}_{J}$ is the reduced Coxeter number of $J$.

(b) If I and J are one-peak principal posets with at most 13 elements and DI, DJ are the associated Euclidean diagrams, then the following conditions are equivalent:

(b1) $D I=D J$,

(b2) $\operatorname{specc}_{I}=\operatorname{specc}_{J}$,

(b3) $\check{\mathbf{c}}_{I}=\check{\mathbf{c}}_{J}$ and $|I|=|J|$,

(b4) the incidence matrix $C_{I} \in \mathbb{M}_{I}(\mathbb{Z})$ is $\mathbb{Z}$-congruent to the incidence matrix $C_{I} \in \mathbb{M}_{I}(\mathbb{Z})$; that is, there is a $\mathbb{Z}$-invertible matrix $B$ such that $C_{I}=B^{t r} \cdot C_{J} \cdot B$.

Proof. (a) By Lemma 8, $\operatorname{specc}_{J} \subseteq \delta^{1}$ and $1 \in \operatorname{specc}_{J}$. Assume that $D J$ is the associated Euclidean diagram of $J$, as in
Proposition 9. By a computer search (using the results of [43] and the inflation algorithm given in $[4,21]$ ), we show that

$$
\operatorname{cox}_{J}(t)=\operatorname{cox}_{D J}(t), \quad \check{\mathbf{c}}_{J}=\check{\mathbf{c}}_{D J},
$$

for any poset $J$, with at most 13 elements. Hence, in view of [4, Proposition 2.17], we have

$$
\operatorname{cox}_{J}(t)=\operatorname{cox}_{D J}(t)=F_{D J}(t)
$$

where

$$
\begin{aligned}
& F_{D J}(t)= \\
& \begin{aligned}
t^{n+1}+t^{n}-t^{n-1}-t^{n-2}-t^{3}-t^{2}+t+1 \\
\quad=(t-1)^{2} \mathfrak{v}_{2}^{2}(t) \mathfrak{v}_{n-2}(t), \quad \text { for } D J=\widetilde{\mathbb{D}}_{n}, \\
t^{7}+t^{6}-2 t^{4}-2 t^{3}+t+1 \\
\quad=(t-1)^{2} \mathfrak{v}_{2}(t) \mathfrak{v}_{3}^{2}(t), \quad \text { for } D J=\widetilde{\mathbb{E}}_{6}, \\
t^{8}+t^{7}-t^{5}-2 t^{4}-t^{3}+t+1 \\
\quad=(t-1)^{2} \mathfrak{v}_{2}(t) \mathfrak{v}_{3}(t) \mathfrak{v}_{4}(t), \quad \text { for } D J=\widetilde{\mathbb{E}}_{7}, \\
t^{9}+t^{8}-t^{6}-t^{5}-t^{4}-t^{3}+t+1 \\
\quad=(t-1)^{2} \mathfrak{v}_{2}(t) \mathfrak{v}_{3}(t) \mathfrak{v}_{5}(t), \quad \text { for } D J=\widetilde{\mathbb{E}}_{8},
\end{aligned}
\end{aligned}
$$

where $\mathfrak{b}_{m}(t)=t^{m-1}+t^{m-2}+t^{m-3}+\cdots+t^{2}+t+1$. For $D J \epsilon$ $\left\{\widetilde{\mathbb{D}}_{4}, \widetilde{\mathbb{D}}_{5}\right\}$, we have

$$
F_{D J}(t)= \begin{cases}t^{5}+t^{4}-2 t^{3}-2 t^{2}+t+1, & \text { for } D J=\widetilde{\mathbb{D}}_{4}, \\ t^{6}+t^{5}-t^{4}-2 t^{3}-t^{2}+t+1, & \text { for } D J=\widetilde{\mathbb{D}}_{5} .\end{cases}
$$

Then, (a) follows by applying [38, Lemma XIII.1.3]. Hence, we also easily conclude that the statements (b1)-(b3) are equivalent.

To finish the proof of (b), we note that the equality $C_{I}=$ $B^{\mathrm{tr}} \cdot C_{J} \cdot B$ in (b4) implies that the matrices $\operatorname{Cox}_{I}$ and $\operatorname{Cox}_{J}$ are conjugate, and, hence, we get $\operatorname{specc}_{I}=$ specc $_{J}$; that is, the implication $(\mathrm{b} 4) \Rightarrow(\mathrm{b} 2)$ holds. To prove the inverse implication (b2) $\Rightarrow(b 4)$, we apply the technique used in [18, Section 6]. On this way, given a principal poset $J$, with at most 13 elements and the associated Euclidean diagram $D J$, we construct (by a computer search) a $\mathbb{Z}$-invertible matrix $B_{J}$ such that $\check{G}_{D J}=B_{J}^{\mathrm{tr}} \cdot C_{J} \cdot B_{J}$ (compare with $\left.[17,18,33,43]\right)$. Hence, (b4) follows, and the proof is complete.

If $J$ is a principal poset, then the sets

$$
\begin{aligned}
& \mathscr{R}_{q_{J}}=\left\{v \in \mathbb{Z}^{m} ; q_{J}(v)=1\right\}, \\
& \mathscr{R}_{\widehat{q}_{J}}=\left\{v \in \mathbb{Z}^{m} ; \hat{q}_{J}(v)=1\right\}, \\
& \mathscr{R}_{\bar{q}_{J}}=\left\{v \in \mathbb{Z}^{m} ; \bar{q}_{J}(v)=1\right\}
\end{aligned}
$$


of roots of the unit forms $q_{J}, \widehat{q}_{J}$, and $\bar{q}_{J}$ have the disjoint union decompositions

$$
\begin{aligned}
& \mathscr{R}_{q_{J}}=\partial_{J}^{-} \mathscr{R}_{q_{J}} \cup \partial_{J}^{+} \mathscr{R}_{q_{J}} \cup \partial_{J}^{0} \mathscr{R}_{q_{J}}, \\
& \mathscr{R}_{\widehat{q}_{J}}=\widehat{\partial}_{J}^{-} \mathscr{R}_{\widehat{q}_{J}} \cup \widehat{\partial}_{J}^{+} \mathscr{R}_{\widehat{q}_{J}} \cup \widehat{\partial}_{J}^{0} \mathscr{R}_{\bar{q}_{J}}, \\
& \mathscr{R}_{\bar{q}_{J}}=\bar{\partial}_{J}^{-} \mathscr{R}_{\bar{q}_{J}} \cup \bar{\partial}_{J}^{+} \mathscr{R}_{\bar{q}_{J}} \cup \bar{\partial}_{J}^{0} \mathscr{R}_{\bar{q}_{J}},
\end{aligned}
$$

where

$$
\begin{aligned}
& \partial_{J}^{-} \mathscr{R}_{q_{J}}=\left\{v \in \mathscr{R}_{q_{J}} ; \partial_{J}(v)<0\right\}, \\
& \partial_{J}^{+} \mathscr{R}_{q_{J}}=\left\{v \in \mathscr{R}_{q_{J}} ; \partial_{J}(v)>0\right\}, \\
& \partial_{J}^{0} \mathscr{R}_{q_{J}}=\left\{v \in \mathscr{R}_{q_{J}} ; \partial_{J}(v)=0\right\} ; \\
& \widehat{\partial}_{J}^{-} \mathscr{R}_{\widehat{q}_{J}}=\left\{v \in \mathscr{R}_{\widehat{q}_{J}} ; \widehat{\partial}_{J}(v)<0\right\}, \\
& \widehat{\partial}_{J}^{+} \mathscr{R}_{\widehat{q}_{J}}=\left\{v \in \mathscr{R}_{\bar{q}_{J}} ; \widehat{\partial}_{J}(v)>0\right\}, \\
& \widehat{\partial}_{J}^{0} \mathscr{R}_{\widehat{q}_{J}}=\left\{v \in \mathscr{R}_{\widehat{q}_{J}} ; \widehat{\partial}_{J}(v)=0\right\} ; \\
& \bar{\partial}_{J}^{-} \mathscr{R}_{\bar{q}_{J}}=\left\{v \in \mathscr{R}_{\bar{q}_{J}} ; \bar{\partial}_{J}(v)<0\right\}, \\
& \bar{\partial}_{J}^{+} \mathscr{R}_{\bar{q}_{J}}=\left\{v \in \mathscr{R}_{\bar{q}_{J}} ; \bar{\partial}_{J}(v)>0\right\}, \\
& \bar{\partial}_{J}^{0} \mathscr{R}_{\bar{q}_{J}}=\left\{v \in \mathscr{R}_{\bar{q}_{J}} ; \bar{\partial}_{J}(v)=0\right\} .
\end{aligned}
$$

Note that the group isomorphism $\mathbb{Z}^{J} \rightarrow \mathbb{Z}^{J}, v \mapsto \widehat{v}:=$ $-v$, restricts to the bijections

$$
\begin{gathered}
\partial_{J}^{-} \mathscr{R}_{q_{J}} \stackrel{\simeq}{\stackrel{\simeq}{\longrightarrow}} \partial_{J}^{+} \mathscr{R}_{q_{J}}, \quad \hat{\partial}_{J}^{-} \mathscr{R}_{\widehat{q}_{J}} \stackrel{\simeq}{\stackrel{\simeq}{\longrightarrow}} \widehat{\partial}_{J}^{+} \mathscr{R}_{\widehat{q}_{J}}, \\
\bar{\partial}_{J} \mathscr{R}_{\bar{q}_{J}} \stackrel{\simeq}{\underset{1-1}{\longrightarrow}} \bar{\partial}_{J}^{+} \mathscr{R}_{\bar{q}_{J}} .
\end{gathered}
$$

Example 12. We compute the reduced Coxeter number, the Coxeter polynomial, and the Euler defect of the following principal two-peak poset

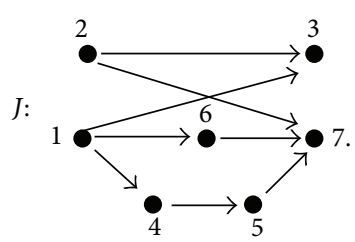

Note that $J$ is principal, because

$$
\begin{aligned}
\bar{q}_{J}(x)= & x_{1}^{2}+x_{2}^{2}+x_{3}^{2}+x_{4}^{2}+x_{5}^{2}+x_{6}^{2} \\
& +x_{7}^{2}-\left(x_{1}+x_{2}\right) x_{3}-\left(x_{1}+x_{5}\right) x_{4} \\
& -x_{1} x_{6}+\left(x_{1}-x_{2}-x_{5}-x_{6}\right) x_{7}
\end{aligned}
$$

$$
\begin{aligned}
= & \left(x_{1}-\frac{1}{2} x_{4}-\frac{1}{2} x_{5}-\frac{1}{2} x_{6}+\frac{1}{2} x_{7}\right)^{2} \\
& +\left(x_{2}-\frac{1}{2} x_{3}-\frac{1}{2} x_{7}\right)^{2} \\
& +\frac{5}{12}\left(x_{3}-\frac{2}{5} x_{5}-\frac{4}{5} x_{6}+\frac{1}{5} x_{7}\right)^{2} \\
& +\frac{3}{4}\left(-\frac{1}{3} x_{3}+x_{4}-\frac{2}{3} x_{5}-\frac{1}{3} x_{6}+\frac{1}{3} x_{7}\right)^{2} \\
& +\frac{3}{5}\left(x_{5}-\frac{1}{2} x_{6}-\frac{1}{2} x_{7}\right)^{2}+\frac{1}{4}\left(x_{6}-x_{7}\right)^{2} .
\end{aligned}
$$

It follows that $\bar{q}_{J}$ is nonnegative and $\operatorname{Ker} \bar{q}_{J}=\mathbb{Z} \cdot \mathbf{h}$, where $\mathbf{h}=(1,1,1,1,1,1,1) ; \bar{q}_{J}$ is critical in the sense of Ovsienko $[24]$; see also $[38,44]$. Note that the Euler matrix $\bar{C}_{J}=C_{J}^{-1}$ of $J$ and the inverse of the Coxeter-Euler matrix $\overline{\operatorname{Cox}}_{J}:=-C_{J}^{-1}$. $C_{J}^{\text {tr }}$ have the forms

$$
\bar{C}_{J}=C_{J}^{-1}=\left[\begin{array}{ccccccc}
1 & 0 & -1 & -1 & 0 & -1 & 1 \\
0 & 1 & -1 & 0 & 0 & 0 & -1 \\
0 & 0 & 1 & 0 & 0 & 0 & 0 \\
0 & 0 & 0 & 1 & -1 & 0 & 0 \\
0 & 0 & 0 & 0 & 1 & 0 & -1 \\
0 & 0 & 0 & 0 & 0 & 1 & -1 \\
0 & 0 & 0 & 0 & 0 & 0 & 1
\end{array}\right]
$$$$
\overline{\operatorname{Cox}}_{J}^{-1}=\left[\begin{array}{ccccccc}
-1 & 0 & -1 & -1 & -1 & -1 & -1 \\
0 & -1 & -1 & 0 & 0 & 0 & -1 \\
1 & 1 & 1 & 1 & 1 & 1 & 2 \\
1 & 0 & 1 & 0 & 0 & 1 & 0 \\
0 & 0 & 0 & 1 & 0 & 0 & 0 \\
1 & 0 & 1 & 1 & 1 & 0 & 0 \\
-1 & 1 & 0 & -1 & 0 & 0 & 1
\end{array}\right] \text {. }
$$

Moreover, we have $G_{\widetilde{\mathbb{E}}_{6}}=B^{\operatorname{tr}} \cdot \bar{G}_{J} \cdot B$, and the matrix $A$ := $B^{\operatorname{tr}} \cdot \bar{C}_{J} \cdot B$ is a morsification of the Euclidean diagram $\widetilde{\mathbb{E}}_{6}$ (see $[34,40])$, where

$$
B=\left[\begin{array}{ccccccc}
-2 & 0 & 1 & 0 & 0 & 0 & 0 \\
0 & 0 & 0 & 0 & 0 & 0 & 1 \\
-1 & 0 & 0 & 0 & 0 & 1 & 0 \\
-1 & 0 & 0 & 0 & 1 & 0 & 0 \\
0 & 0 & 0 & 1 & 0 & 0 & 0 \\
-1 & 1 & 0 & 0 & 0 & 0 & 0 \\
1 & 0 & 0 & 0 & 0 & 0 & 0
\end{array}\right]
$$

$$
A=\left[\begin{array}{ccccccc}
1 & 1 & -2 & 1 & 1 & 1 & 0 \\
-2 & 1 & 0 & 0 & 0 & 0 & 0 \\
2 & -1 & 1 & 0 & -1 & -1 & 0 \\
-1 & 0 & 0 & 1 & 0 & 0 & 0 \\
-1 & 0 & 0 & -1 & 1 & 0 & 0 \\
-1 & 0 & 0 & 0 & 0 & 1 & 0 \\
0 & 0 & 0 & 0 & 0 & -1 & 1
\end{array}\right] \text {. }
$$


Hence, in view of [2, Proposition 2.8], we get the following:

(i) the Euclidean type $D J$ of $J$ is the diagram $\widetilde{\mathbb{E}}_{6}$, and the Coxeter polynomial of the poset $J$ has the form

$$
\operatorname{cox}_{J}(t)=t^{7}-t^{5}-t^{2}+1=\operatorname{cox}_{A}(t)
$$

that is, $\operatorname{cox}_{J}(t)$ is the Coxeter polynomial $F_{\Delta}^{(2)}(t)(21)$, of the Euclidean diagram $\Delta=\widetilde{\mathbb{A}}_{6}$ (with a particular numbering of vertices), and is the Coxeter polynomial $\operatorname{cox}_{A}(t)$ of the morsification $A \in \mathbb{M}_{7}(\mathbb{Z})$ of the $\operatorname{diagram} \widetilde{\mathbb{E}}_{6}$,

(ii) the Coxeter number $\mathbf{c}_{J}$ is infinite and the reduced Coxeter number $\check{\mathbf{c}}_{J}$ equals 10 ,

(iii) the Euler defect has the form $\bar{\partial}_{J}(x)=3\left(x_{1}+x_{2}-x_{3}-\right.$ $\left.x_{7}\right)$

(iv) the $\bar{\Phi}_{J}$-orbit of any vector of defect zero in $\bar{\partial}_{J}^{0} \mathscr{R}_{\widehat{q}_{J}}$ is of length 2 or of length 5 . It is shown in [1, Remark 4.5] and [34, Example 5.14] that they lie on one sand-glass tube $\mathscr{T}_{1,2}$ of rank 2 and on six sand-glass tubes of rank five.

\section{Nonnegative Posets of Positive Corank}

In the study of nonnegative posets, the following extensions of [34, Definition 2.2] are of importance.

Definition 13. Assume that $m \geq 2, r \geq 0$, and $q: \mathbb{Z}^{m} \rightarrow \mathbb{Z}$ is a unit quadratic form.

(a) The form $q$ is defined to be nonnegative of corank $r \geq$ 0 , if $q$ is nonnegative and the $\mathbb{Q}$-rank $\operatorname{rk}_{\mathbb{Q}} G_{q}$ of the rational Gram matrix $G_{q} \in \mathbb{M}_{m}(\mathbb{Q})$ equals $\operatorname{rk}_{\mathbb{Q}} G_{q}=$ $m-r$.

(b) The form $q$ is defined to be nonnegative critical of corank $r \geq 1$, if $q$ is nonnegative of corank $r \geq 1$ and each of the nonnegative quadratic forms $q^{(1)}, \ldots, q^{(m)}: \mathbb{Z}^{m-1} \rightarrow \mathbb{Z}$ is of corank at most $r-1 \geq 0$, where

$$
\begin{aligned}
& q^{(j)}\left(x_{1}, \ldots, x_{j-1}, x_{j+1}, \ldots, x_{m}\right) \\
& \quad=q\left(x_{1}, \ldots, x_{j-1}, 0, x_{j+1}, \ldots, x_{m}\right) .
\end{aligned}
$$

Lemma 14. Assume that $m \geq 2, r \geq 0$, and $q: \mathbb{Z}^{m} \rightarrow \mathbb{Z}$ is an integral quadratic form.

(a) $q$ is nonnegative of corank $r \geq 0$ if and only if $q$ is nonnegative and the subgroup Ker $q$ of the abelian group $\mathbb{Z}^{m}$ is free of rank $r$. (b) $q$ is nonnegative of corank $r=0$ if and only if $q$ is positive, and $q$ is nonnegative of corank one if and only if $q$ is principal.

(c) $q$ is nonnegative critical of corank $r \geq 1$ if and only if $q$ is nonnegative and, for any $j \in\{1, \ldots, m\}$, the abelian subgroup $\mathbb{Z}^{m, j} \cap \operatorname{Ker} q$ of $\mathbb{Z}^{m}$ is free of rank at most $r-1$, where

$$
\begin{aligned}
\mathbb{Z}^{m, j} & :=\mathbb{Z}^{m-j-1} \times\{0\} \times \mathbb{Z}^{j-1} \\
& =\left\{v=\left(v_{1}, \ldots, v_{m}\right) \in \mathbb{Z}^{m} ; v_{j}=0\right\} \subseteq \mathbb{Z}^{m}
\end{aligned}
$$

is viewed as a subgroup of $\mathbb{Z}^{m}$.

(d) $q$ is nonnegative critical of corank $r=1$ if and only if $q$ is P-critical in the sense of [34, Definition 2.2] and [44].

Proof. The proof of (a) follows by applying the arguments used in the proof of the equivalence $(a) \Leftrightarrow(b)$ in Proposition 9. The statements (b) and (c) follow from (a).

(c) First, we note that the quadratic forms $q^{(1)}, \ldots, q^{(m)}$ : $\mathbb{Z}^{m-1} \rightarrow \mathbb{Z}$ are nonnegative, if $q$ is nonnegative. Then, (c) is a consequence of the group isomorphism

$$
\begin{gathered}
\operatorname{Ker} q^{(j)} \stackrel{\simeq}{\longrightarrow} \mathbb{Z}^{m, j} \cap \operatorname{Ker} q, \\
w \longmapsto \widehat{w}^{(j)}:=\left(w_{1}, \ldots, w_{j-1}, 0, w_{j+1}, \ldots, w_{m}\right) .
\end{gathered}
$$

Since (d) is a consequence of (c), the proof is complete.

Definition 15. Assume that $J$ is a connected poset and $q_{J}, \widehat{q}_{J}$ : $\mathbb{Z}^{J} \rightarrow \mathbb{Z}$ are its incidence and Tits quadratic forms (6), respectively.

(a) $J$ is defined to be nonnegative of corank $r \geq 0$ if its incidence quadratic form $q_{J}: \mathbb{Z}^{J} \rightarrow \mathbb{Z}$ (resp., one of the forms $\hat{q}_{J}$ and $\bar{q}_{J}$ ) is nonnegative and the free abelian subgroup Ker $q_{J}$ of $\mathbb{Z}^{J}$ is of $\mathbb{Z}$-rank $r$ (resp., $\operatorname{Ker} \widehat{q}_{J} \cong \operatorname{Ker} \bar{q}_{J} \cong \operatorname{Ker} q_{J}$ is of $\mathbb{Z}$-rank $r$ ); see (36).

(b) $J$ is defined to be nonprincipal critical if the incidence quadratic form $q_{J}: \mathbb{Z}^{J} \rightarrow \mathbb{Z}$ is nonnegative and not positive, $J$ is not principal, and the quadratic form $q_{J^{\prime}}: \mathbb{Z}^{J^{\prime}} \rightarrow \mathbb{Z}$ is principal or positive, for every proper subposet $J^{\prime}$ of $J$.

(c) A one-peak poset $I$, with $\max I=\{*\}$, is defined to be nonprincipal Tits-critical if the Tits quadratic form $\hat{q}_{I}: \mathbb{Z}^{I} \rightarrow \mathbb{Z}$ is nonnegative and not positive, $I$ is not principal, and the Tits quadratic form $\widehat{q}_{I^{\prime}}: \mathbb{Z}^{I^{\prime}} \rightarrow \mathbb{Z}$ is principal or positive, for every proper subposet $I^{\prime}$ of 
I containing the peak $*$. We call a nonprincipal Titscritical poset $I$ exceptional, if the subposet $T=I \backslash\{*\}$ is nonprincipal Tits-critical; see [33, 34].

(d) A poset $J$ is defined to be P-hypercritical if $J$ is not nonnegative and each of its proper subposet is nonnegative; see [34, Definition 2.2].

Remark 16. Assume that $T$ is a poset and $T^{*}=T \cup\{*\}$ is its one-peak enlargement.

(a) If $T^{*}$ is $P$-hypercritical, then $T$ is $N P$-critical in the sense of [14], but not conversely.

(b) By [43], many of the NP-critical posets $T$ listed in [14, Table 2] are of corank at most two.

(c) A Coxeter spectral classification of one-peak positive (resp., almost Tits $P$-critical) posets is given in $[17,18]$ (resp., in [33]). tion.

We frequently use the following important characterisa-

Theorem 17. Assume that $J$ is a connected poset and $q_{J}, \widehat{q}_{J}$ : $\mathbb{Z}^{J} \rightarrow \mathbb{Z}$ are the incidence and the Tits quadratic forms of $J$ (7), respectively.

(a) If $J$ is nonnegative of corank two, then J contains at least 6 elements, and $|J|=6$ if and only if $J$ is the garland

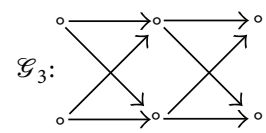

with $\operatorname{cox}_{\mathscr{G}_{3}}(t)=t^{6}+2 t^{5}-t^{4}-4 t^{3}-t^{2}+2 t+1$

and $\operatorname{Ker} q_{\mathscr{C}_{3}}:=\mathbb{Z} \cdot \mathbf{h}^{(1)} \oplus \mathbb{Z} \cdot \mathbf{h}^{(2)}$, where $\mathbf{h}^{(1)}=$ $(1,1,-1,-1,0,0)$ and $\mathbf{h}^{(2)}=(1,1,0,0,-1,-1)$. The garland $\mathscr{G}_{3}$ is nonprincipal critical.

(b) The following four conditions are equivalent.

(b1) The poset J is nonprincipal critical.

(b2) $|J| \geq 6$ and the form $q_{J}: \mathbb{Z}^{J} \rightarrow \mathbb{Z}$ is nonnegative critical of corank two.

(b3) $|J| \geq 6$ and $q_{J}: \mathbb{Z}^{J} \rightarrow \mathbb{Z}$ is nonnegative, the group $\operatorname{Ker} \mathrm{q}_{J}$ is of $\mathbb{Z}$-rank two, and for any $j \in J$, the subposet $J^{(j)}:=J \backslash\{j\}$ of $J$ is principal or positive.

(b4) $|J| \geq 6$ and $q_{J}: \mathbb{Z}^{J} \rightarrow \mathbb{Z}$ is nonnegative, and the group Ker $q_{J}$ has a $\mathbb{Z}$-basis $\mathbf{h}, \mathbf{h}^{\prime}$ such that there is no $j \in J$, with $h_{j}=h_{j}^{\prime}=0$. (c) Let I be a one-peak poset $I$, with $\max I=\{*\}$. The following three conditions are equivalent.

(c1) I is nonprincipal Tits-critical.

(c2) $|I| \geq 7$, the Tits form $\widehat{q}_{I}: \mathbb{Z}^{I} \rightarrow \mathbb{Z}$ is nonnegative, the group Ker $\widehat{q}_{I}$ is of $\mathbb{Z}$-rank two, and for any $j \in I \backslash\{*\}$, the one-peak subposet $I^{(j)}:=I \backslash\{j\}$ of $I$ is principal or positive.

(c3) $|I| \geq 7$ and $\widehat{q}_{I}: \mathbb{Z}^{I} \rightarrow \mathbb{Z}$ is nonnegative, and the group $\operatorname{Ker} \widehat{q}_{I}$ has a $\mathbb{Z}$-basis $\mathbf{h}, \mathbf{h}^{\prime}$ such that there is no $j \in I \backslash\{*\}$, with $h_{j}=h_{j}^{\prime}=0$.

(d) A nonprincipal Tits-critical one-peak poset I, with $\max I=\{*\}$ and $|I|=7$, is exceptional if and only if I is the one-peak garland

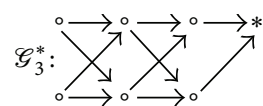

$$
\text { with } \operatorname{cox}_{\mathscr{G}_{3}^{*}}(t)=t^{7}+t^{6}-t^{5}-t^{4}-t^{3}-t^{2}+t+1
$$

and $\operatorname{Ker} \widehat{q}_{\mathscr{E}_{3}^{*}}:=\mathbb{Z} \cdot \widehat{\mathbf{h}}^{(1)} \oplus \mathbb{Z} \cdot \widehat{\mathbf{h}}^{(2)}$, where $\widehat{\mathbf{h}}^{(1)}=$ $(1,1,-1,-1,0,0,0), \widehat{\mathbf{h}}^{(2)}=(1,1,0,0,-1,-1,0)$.

Proof. (a) It is easy to check that any poset $J$ with at most 5 elements is either positive or principal. Moreover, if $J$ is nonnegative of corank two and $|J|=6$, then $J$ is the garland $\mathscr{G}_{3}$. Since

$$
\begin{aligned}
q_{\mathscr{G}_{3}}(x)= & x_{1}^{2}+x_{2}^{2}+x_{3}^{2}+x_{4}^{2}+x_{5}^{2}+x_{6}^{2} \\
& +\left(x_{1}+x_{2}\right)\left(x_{3}+x_{4}+x_{5}+x_{6}\right) \\
& +\left(x_{3}+x_{4}\right)\left(x_{5}+x_{6}\right),
\end{aligned}
$$

the Lagrange's algorithm yields

$$
\begin{aligned}
q_{\mathscr{S}_{3}}(x)= & \left(x_{1}+\frac{1}{2} x_{3}+\frac{1}{2} x_{4}+\frac{1}{2} x_{5}+\frac{1}{2} x_{6}\right)^{2} \\
& +\left(x_{2}+\frac{1}{2} x_{3}+\frac{1}{2} x_{4}+\frac{1}{2} x_{5}+\frac{1}{2} x_{6}\right)^{2} \\
& +\frac{1}{2}\left(x_{3}-x_{4}\right)^{2}+\frac{1}{2}\left(x_{5}-x_{6}\right)^{2} .
\end{aligned}
$$

It follows that $q_{\mathscr{G}_{3}}: \mathbb{Z}^{6} \rightarrow \mathbb{Z}$ is nonnegative and its kernel is a rank-two free abelian group of the form shown in (a). Hence, (a) follows.

(b) We show by a computer search that there is no nonprincipal critical poset $J$ such that $|J| \leq 5$. Then, in view of Lemma 14, the equivalences (b1) $\Leftrightarrow(\mathrm{b} 2) \Leftrightarrow(\mathrm{b} 3) \Leftrightarrow(\mathrm{b} 4)$ easily follow.

(c) We show by a computer search that there is no onepeak nonprincipal Tits-critical poset $I$ such that $|I| \leq 6$. Then, in view of Lemma 14 , the equivalences $(\mathrm{c} 1) \Leftrightarrow(\mathrm{c} 2) \Leftrightarrow(\mathrm{c} 3)$ easily follow. 
(d) Note that

$$
\begin{aligned}
\widehat{q}_{\mathscr{G}_{3}^{*}}(x)= & x_{1}^{2}+x_{2}^{2}+x_{3}^{2}+x_{4}^{2}+x_{5}^{2}+x_{6}^{2}+x_{7}^{2} \\
& +\left(x_{1}+x_{2}\right)\left(x_{3}+x_{4}+x_{5}+x_{6}\right) \\
& +\left(x_{3}+x_{4}\right)\left(x_{5}+x_{6}\right) \\
& -\left(x_{1}+x_{2}+x_{3}+x_{4}+x_{5}+x_{6}\right) x_{7},
\end{aligned}
$$

and the Lagrange's algorithm yields

$$
\begin{aligned}
\widehat{q}_{\mathscr{S}_{3}^{*}}(x)= & \left(x_{1}+\frac{1}{2} x_{3}+\frac{1}{2} x_{4}+\frac{1}{2} x_{5}+\frac{1}{2} x_{6}-\frac{1}{2} x_{7}\right)^{2} \\
& +\left(x_{2}+\frac{1}{2} x_{3}+\frac{1}{2} x_{4}+\frac{1}{2} x_{5}+\frac{1}{2} x_{6}-\frac{1}{2} x_{7}\right)^{2} \\
& +\frac{1}{2}\left(x_{3}-x_{4}\right)^{2}+\frac{1}{2}\left(x_{5}-x_{6}\right)^{2}+\frac{1}{2} x_{7}^{2} .
\end{aligned}
$$

It follows that $\widehat{q}_{\mathscr{S}_{3}^{*}}: \mathbb{Z}^{7} \rightarrow \mathbb{Z}$ is nonnegative and its kernel is a rank-two free abelian group of the form shown in (d). Hence, the one-peak garland $\mathscr{G}_{3}^{*}$ is nonprincipal Tits-critical and exceptional. On the other hand, one shows by a computer search that $\mathscr{G}_{3}^{*}$ is the only one-peak poset that is nonprincipal Tits-critical and exceptional. This finishes the proof.

Following [34, Section 4], we will study nonnegative posets $J$ of corank $r \geq 2$ by means of the spectrum specc $_{J}$, the reduced Coxeter number $\check{\mathbf{c}}_{J}$, and the rank $r \geq 2$ defects

$$
\begin{gathered}
\partial_{J}=\left(\partial_{J}^{(1)}, \ldots, \partial_{J}^{(r)}\right), \quad \bar{\partial}_{J}=\left(\bar{\partial}_{J}^{(1)}, \ldots, \bar{\partial}_{J}^{(r)}\right), \\
\widehat{\partial}_{J}=\left(\widehat{\partial}_{J}^{(1)}, \ldots, \widehat{\partial}_{J}^{(r)}\right): \mathbb{Z}^{J} \longrightarrow \mathbb{Z}^{r}
\end{gathered}
$$

defined in the following extension of Theorem 10.

Theorem 18. Let $J$ be a finite nonnegative poset of corank $r \geq$ 2 , and let $m=|J| \geq 2$. One fixes nonzero vectors $\mathbf{h}_{J}^{(1)}, \ldots, \mathbf{h}_{J}^{(r)} \in$ $\mathbb{Z}^{J}$ such that $\operatorname{Ker} q_{J}=\mathbb{Z} \cdot \mathbf{h}_{J}^{(1)} \oplus \cdots \oplus \mathbb{Z} \cdot \mathbf{h}_{J}^{(r)} \cong \mathbb{Z}^{r}$, and one sets $\mathbf{h}_{J}=\left(\mathbf{h}_{J}^{(1)}, \ldots, \mathbf{h}_{J}^{(r)}\right)$.

(a) There exist a minimal integer $\check{\mathbf{c}}_{J} \geq 1$ (called the reduced Coxeter number of $J$ ) and a group homomorphism $\partial_{J}=\left(\partial_{J}^{(1)}, \ldots, \partial_{J}^{(r)}\right): \mathbb{Z}^{J} \rightarrow \mathbb{Z}^{r} \cong \operatorname{Ker} q_{J}$ (called the incidence defect of $J$ ) such that

$$
\begin{aligned}
\Phi_{J}^{\check{c}_{J}}(v) & =v+\partial_{J}(v) \cdot \mathbf{h}_{J} \\
& =v+\partial_{J}^{(1)}(v) \cdot \mathbf{h}_{J}^{(1)}+\cdots+\partial_{J}^{(r)}(v) \cdot \mathbf{h}_{J}^{(r)}, \\
& \partial_{J}\left(\Phi_{J}(v)\right)=\partial_{J}(v), \quad \forall v \in \mathbb{Z}^{J},
\end{aligned}
$$

and $\partial_{J}(\mathbf{h})=0$, for all $\mathbf{h} \in \operatorname{Ker} q_{J}$, where one sets

$$
\begin{gathered}
\partial_{J}(v)=\left(\partial_{J}^{(1)}(v), \ldots, \partial_{J}^{(r)}(v)\right), \\
\partial_{J}(v) \cdot \mathbf{h}_{J}:=\partial_{J}^{(1)}(v) \cdot \mathbf{h}_{J}^{(1)}+\cdots+\partial_{J}^{(r)}(v) \cdot \mathbf{h}_{J}^{(r)} .
\end{gathered}
$$

(b) Assume that $\check{\mathbf{c}}_{J} \geq 1$ and $\partial_{J}: \mathbb{Z}^{J} \rightarrow \mathbb{Z}^{r}$ are as in (a), and one sets

$$
\begin{gathered}
\overline{\mathbf{h}}_{J}^{(1)}=\mathbf{h}_{J}^{(1)} \cdot B^{\prime}, \ldots, \overline{\mathbf{h}}_{J}^{(r)}=\mathbf{h}_{J}^{(r)} \cdot B^{\prime}, \\
\widehat{\mathbf{h}}_{J}^{(1)}=\mathbf{h}_{J}^{(1)} \cdot B^{\prime} \cdot B^{-1}, \ldots, \widehat{\mathbf{h}}_{J}^{(r)}=\mathbf{h}_{J}^{(r)} \cdot B^{\prime} \cdot B^{-1},
\end{gathered}
$$

where $B^{\prime}, B \in \mathbb{M}_{J}(\mathbb{Z})$ are as in Proposition 5.

(b1) There exists a group homomorphism $\bar{\partial}_{J}=$ $\left(\bar{\partial}_{J}^{(1)}, \ldots, \bar{\partial}_{J}^{(r)}\right): \mathbb{Z}^{J} \rightarrow \mathbb{Z}^{r} \cong \operatorname{Ker} \bar{q}_{J}$ (called the Euler defect of $J$ ) such that

$$
\begin{array}{r}
\bar{\Phi}_{J}^{\check{\mathfrak{c}}_{J}}(v)=v+\bar{\partial}_{J}(v) \cdot \overline{\mathbf{h}}_{J} \\
=v+\bar{\partial}_{J}^{(1)}(v) \cdot \overline{\mathbf{h}}_{J}^{(1)}+\cdots+\bar{\partial}_{J}^{(r)}(v) \cdot \overline{\mathbf{h}}_{J}^{(r)}, \\
\forall v \in \mathbb{Z}^{J},
\end{array}
$$

$\bar{\partial}_{J} \circ \bar{\Phi}_{I}=\bar{\partial}_{I}, \bar{\partial}_{J}=\bar{\partial}_{J} \circ h_{B^{\prime}}$, and $\bar{\partial}_{J}(\mathbf{h})=0$, for all $\mathbf{h} \in \operatorname{Ker} \bar{q}_{J}$, where one sets

$$
\overline{\mathbf{h}}_{J}=\left(\overline{\mathbf{h}}_{J}^{(1)}, \ldots, \overline{\mathbf{h}}_{J}^{(r)}\right),
$$

$$
\bar{\partial}_{J}(v) \cdot \overline{\mathbf{h}}_{J}:=\bar{\partial}_{J}^{(1)}(v) \cdot \overline{\mathbf{h}}_{J}^{(1)}+\cdots+\bar{\partial}_{J}^{(r)}(v) \cdot \overline{\mathbf{h}}_{J}^{(r)} .
$$

(b2) There exists a group homomorphism $\widehat{\partial}_{J}=$ $\left(\widehat{\partial}_{J}^{(1)}, \ldots, \widehat{\partial}_{J}^{(r)}\right): \mathbb{Z}^{J} \rightarrow \mathbb{Z}^{r} \cong \operatorname{Ker} \widehat{q}_{J}$ (called the Tits defect of $J$ ) such that

$$
\begin{aligned}
\widehat{\Phi}_{J}^{\check{c}_{J}}(v) & =v+\widehat{\partial}_{J}(v) \cdot \widehat{\mathbf{h}}_{J} \\
& =v+\bar{\partial}_{J}^{(1)}(v) \cdot \widehat{\mathbf{h}}_{J}^{(1)}+\cdots+\widehat{\partial}_{J}^{(r)}(v) \cdot \widehat{\mathbf{h}}_{J}^{(r)},
\end{aligned}
$$

$\forall v \in \mathbb{Z}^{J}$,

$\widehat{\partial}_{J} \circ \widehat{\Phi}_{J}=\widehat{\partial}_{J}, \widehat{\partial}_{J}=\bar{\partial}_{J} \circ h_{B}=\partial_{J} \circ h_{B^{\prime}}^{-1} \circ h_{B}$, and $\widehat{\partial}_{J}(\mathbf{h})=0$, for all $\mathbf{h} \in \operatorname{Ker} \widehat{q}_{\text {J }}$, where one sets

$$
\widehat{\mathbf{h}}_{J}=\left(\widehat{\mathbf{h}}_{J}^{(1)}, \ldots, \widehat{\mathbf{h}}_{J}^{(r)}\right),
$$

$$
\widehat{\partial}_{J}(v) \cdot \widehat{\mathbf{h}}_{J}:=\widehat{\partial}_{J}^{(1)}(v) \cdot \widehat{\mathbf{h}}_{J}^{(1)}+\cdots+\widehat{\partial}_{J}^{(r)}(v) \cdot \widehat{\mathbf{h}}_{J}^{(r)} .
$$

(c) The Coxeter number $\mathbf{c}_{J}$ of $J$ is finite if and only if the incidence defect $\partial_{J}: \mathbb{Z}^{J} \rightarrow \mathbb{Z}^{r}$ is zero. In this case, $\check{\mathbf{c}}_{J}=\mathbf{c}_{J}$.

(d) Given $v \in \mathbb{Z}^{m} \equiv \mathbb{Z}^{J}$, the order $\mathbf{s}_{v}:=|\mathcal{O}(v)|$ of the $\Phi_{J}$-orbit $\mathcal{O}(v)$ is finite if and only if $\partial_{J}(v)=0$. If $\mathbf{s}_{v}=$ $|\mathcal{O}(v)|$ is finite, then $\mathbf{s}_{v}$ divides $\check{\mathbf{c}}_{J}$ and there is a unique integer $m_{v}$ such that

$$
\begin{aligned}
m_{v} \cdot \mathbf{h} & =v+\Phi_{J}(v)+\Phi_{J}^{2}(v)+\cdots+\Phi_{J}^{\mathbf{s}_{v}-1}(v) \\
& =v+\Phi_{J}^{-1}(v)+\Phi_{J}^{-2}(v)+\cdots+\Phi_{J}^{-\mathbf{s}_{v}+1}(v) .
\end{aligned}
$$


(e) The statement (d) holds with $\Phi_{J}$ and $\widehat{\Phi}_{J}$ (resp., $\bar{\Phi}_{J}$ ) interchanged.

Proof. For simplicity of presentation, we assume that $r=2$. We recall from the proof of Proposition 9 that $\mathbb{Z} \cdot \mathbf{h}_{J}^{(1)} \oplus \mathbb{Z}$. $\mathbf{h}_{J}^{(2)}=\operatorname{Ker} q_{J}=\operatorname{Ker}\left[D q_{J}: \mathbb{Z}^{m} \rightarrow \mathbb{Z}^{m}\right]$, where $m=|J| \geq 2$ and

$$
\begin{gathered}
D q_{J}: \mathbb{Z}^{m} \longrightarrow \mathbb{Z}^{m}, \\
v \longmapsto D q_{J}(v)=\left(\frac{\partial q_{J}}{\partial x_{1}}(v), \ldots, \frac{\partial q_{J}}{\partial x_{m}}(v)\right),
\end{gathered}
$$

is the gradient group homomorphism. It follows that

$$
\frac{\mathbb{Z}^{m}}{\mathbb{Z} \cdot \mathbf{h}_{J}^{(1)} \oplus \mathbb{Z} \cdot \mathbf{h}_{J}^{(2)}}=\frac{\mathbb{Z}^{m}}{\operatorname{Ker} q_{J}} \cong \operatorname{Im} D q_{J} \cong \mathbb{Z}^{m-2} .
$$

Denote by $\phi: \mathbb{Z}^{m} \rightarrow \mathbb{Z}^{m-2}$ the composite quotient epimorphism. Then, the form $q_{J}$ induces the form $\widetilde{q}_{J}$ : $\mathbb{Z}^{m-2} \rightarrow \mathbb{Z}$ such that $\tilde{q}_{J}(\phi(x))=q_{J}(x)$, for all $x \in \mathbb{Z}^{m}$. Moreover, the Coxeter transformation $\Phi_{J}: \mathbb{Z}^{m} \rightarrow \mathbb{Z}^{m}$ induces a group automorphism $\widetilde{\Phi}_{J}: \mathbb{Z}^{m-2} \rightarrow \mathbb{Z}^{m-2}$ such that

$$
\widetilde{\Phi}_{J} \circ \phi=\phi \circ \Phi_{J}, \quad \widetilde{q}_{J}\left(\widetilde{\Phi}_{J}(y)\right)=\widetilde{q}_{J}(y),
$$

for all $y \in \mathbb{Z}^{m-2}$. It follows that $\tilde{q}_{J}$ is positive definite, and there exists a minimal integer $\check{\mathbf{c}}_{J} \geq 1$ such that $\widetilde{\Phi}_{J}^{\check{\mathbf{c}}_{J}}$ is the identity map on $\mathbb{Z}^{m-2}$. Hence, given $v \in \mathbb{Z}^{m}$, the element $\Phi_{J}^{\check{c}_{J}}(v)-v$ lies in the kernel of $q_{J}$; that is, it has the form

$$
\Phi_{J}^{\check{\mathbf{c}}_{J}}(v)-v=\partial_{J}^{(1)}(v) \cdot \mathbf{h}_{J}^{(1)}+\partial_{J}^{(2)}(v) \cdot \mathbf{h}_{J}^{(2)}
$$

where $\partial_{J}^{(1)}(v), \partial_{J}^{(2)}(v)$ are integers uniquely determined by $v$. Since $\Phi_{J}$ is a group homomorphism, then

$$
\begin{aligned}
& \partial_{J}^{(1)}\left(v+v^{\prime}\right)=\partial_{J}^{(1)}(v)+\partial_{J}^{(1)}\left(v^{\prime}\right), \\
& \partial_{J}^{(2)}\left(v+v^{\prime}\right)=\partial_{J}^{(2)}(v)+\partial_{J}^{(2)}\left(v^{\prime}\right) ;
\end{aligned}
$$

that is, we have defined a pair of group homomorphisms $\partial_{J}^{(1)}, \partial_{J}^{(2)}: \mathbb{Z}^{J} \rightarrow \mathbb{Z}$; hence, $\partial_{J}=\left(\partial_{J}^{(1)}, \partial_{J}^{(2)}\right): \mathbb{Z}^{J} \rightarrow \mathbb{Z}^{2}$ is a group homomorphism. It is easy to see that $\partial_{J}$ has the properties required in (a), and (a) follows.

In view of Proposition 5, the statements (b)-(e) are a consequence of (a). The reader is referred to [34, Theorem 4.17] for more details and a generalization.

Corollary 19. Assume that $J$ is a finite nonnegative poset of corank $r \geq 2$.

(a) The Coxeter number $\mathbf{c}_{J}$ of $J$ is infinite if and only if the defect homomorphism $\partial_{J}: \mathbb{Z}^{J} \rightarrow \mathbb{Z}^{r}$ is nonzero, or, equivalently, if and only if the $\Phi_{J}$-orbit $\mathcal{O}\left(e_{j}\right)$ of some basis vector $e_{j} \in \mathbb{Z}^{J}$ is infinite.

(b) The Coxeter transformation $\Phi_{J}$ is weakly periodic in the sense of Sato [42]; that is, $\Phi_{J}^{s}-$ id is nilpotent, for some $s \geq 1$.
Proof. The statement (a) follows immediately from Theorem 18. To prove (b), we check that $\left(\Phi_{J}^{\check{\mathbf{c}}_{J}}-i d\right)^{2}=0$.

Remark 20. (a) It was shown in [34, Example 5.18] that, for the one-peak garland $I=\mathscr{G}_{3}^{*}$ of Theorem 17(d), we have

(i) $\partial_{I}=\widehat{\partial}_{I}=\bar{\partial}_{I}=0$ and $\mathbf{c}_{I}=\check{\mathbf{c}}_{I}=4$,

(ii) the set $\mathscr{R}_{\widehat{q}_{I}}$ of Tits roots of $I$ lies on 22 sand-glass tubes; six of them are of rank two, and each of the remaining fourteen tubes is of rank four; see [34, pp. 459-461] for details.

(b) By Lemma $8(\mathrm{a})$, the Coxeter number $\mathrm{c}_{J}$ is infinite, for every principal poset $J$.

(c) By Theorem 17, there is no nonnegative connected poset $J$ of corank 2 , with $|J| \leq 5$. Moreover, a minimal such a poset is the garland

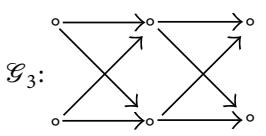

(d) We show in [43] that most of the nonnegative connected posets $J$ of corank 2 , with at most 15 elements, are of zero defect. We also show there that a smallest nonnegative connected poset $J$ with nonzero defect has 8 elements and is one of the following two posets:
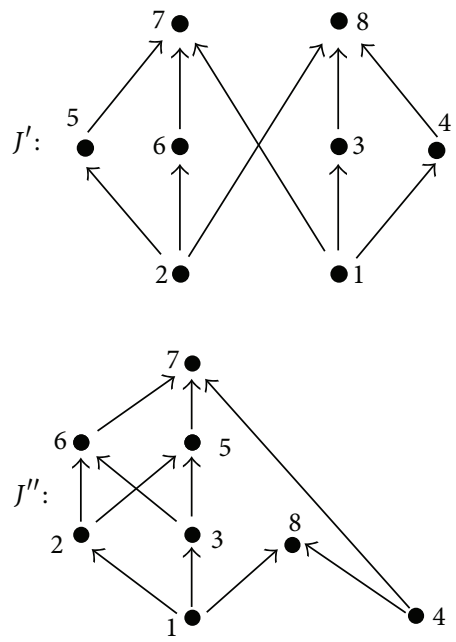

It is easy to check that

(i) $\check{\mathbf{c}}_{J^{\prime}}=\check{\mathbf{c}}_{J^{\prime \prime}}=2$,

(ii) $\operatorname{cox}_{J^{\prime}}(t)=\operatorname{cox}_{J^{\prime \prime}}(t)=t^{8}-4 t^{6}+6 t^{4}-4 t^{2}+1$,

(iii) the coordinates of the Tits defect $\widehat{\partial}_{J^{\prime}}=\left(\widehat{\partial}_{J^{\prime}}^{(1)}, \widehat{\partial}_{J^{\prime}}^{(2)}\right)$ : $\mathbb{Z}^{8} \rightarrow \mathbb{Z}^{2}$ of $J^{\prime}$, with respect to the $\mathbb{Z}$-basis

$$
\begin{aligned}
\widehat{\mathbf{h}}_{J^{\prime}}^{(1)} & =(2,0,-1,-1,1,1,2,0), \\
\widehat{\mathbf{h}}_{J^{\prime}}^{(2)} & =(0,2,1,1,-1,-1,0,2)
\end{aligned}
$$


of $\operatorname{Ker} \widehat{q}_{J^{\prime}}$, are given by the formulae

$$
\begin{aligned}
& \widehat{\partial}_{J^{\prime}}^{(1)}(x)=x_{2}-\frac{1}{2} x_{3}-\frac{1}{2} x_{4}+\frac{1}{2} x_{5}+\frac{1}{2} x_{6}-x_{7}, \\
& \widehat{\partial}_{J^{\prime}}^{(2)}(x)=x_{1}+\frac{1}{2} x_{3}+\frac{1}{2} x_{4}-\frac{1}{2} x_{5}-\frac{1}{2} x_{6}-x_{8},
\end{aligned}
$$

(iv) the coordinates of the Tits defect $\widehat{\partial}_{J^{\prime \prime}}=\left(\widehat{\partial}_{J^{\prime \prime}}^{(1)}, \widehat{\partial}_{J^{\prime \prime}}^{(2)}\right)$ : $\mathbb{Z}^{8} \rightarrow \mathbb{Z}^{2}$ of $J^{\prime \prime}$, with respect to the $\mathbb{Z}$-basis

$$
\begin{gathered}
\widehat{\mathbf{h}}_{J^{\prime \prime}}^{(1)}=(0,-1,-1,0,1,1,0,0), \\
\widehat{\mathbf{h}}_{J^{\prime \prime}}^{(2)}=(1,0,0,1,0,0,1,1)
\end{gathered}
$$

of $\operatorname{Ker} \widehat{q}_{J^{\prime \prime}}$, are given by

$$
\begin{gathered}
\widehat{\partial}_{J^{\prime}}^{(1)}(x)=x_{1}+x_{2}+x_{3}+x_{4}+x_{5}+x_{6}-x_{7}-x_{8}, \\
\widehat{\partial}_{J^{\prime \prime}}^{(2)}(x)=2 x_{1}+x_{2}+x_{3}+2 x_{4}+x_{5}+x_{6}-2 x_{7}-2 x_{8} .
\end{gathered}
$$

\section{An Example}

In this section, we illustrate the results of Section 3 by an example of a principal one-peak poset $I$ of the Euclidean type $D I=\widetilde{\mathbb{D}}_{4}$. We give a description of the set $\mathscr{R}_{\widehat{q}_{I}}$ of roots of $\widehat{q}_{I}$ and the mesh translation quiver $\Gamma\left(\mathscr{R}_{\widehat{q}_{I}}, \widehat{\Phi}_{I}\right)$ together with the decomposition (see (51))

$$
\begin{aligned}
& \Gamma\left(\mathscr{R}_{\widehat{q}_{I}}, \widehat{\Phi}_{I}\right)=\Gamma\left(\widehat{\partial}_{I}^{-} \mathscr{R}_{\widehat{q}_{I}}, \widehat{\Phi}_{I}\right) \cup \Gamma\left(\widehat{\partial}_{I}^{+} \mathscr{R}_{\widehat{q}_{I}}, \widehat{\Phi}_{I}\right) \\
& \cup \Gamma\left(\widehat{\partial}_{I}^{0} \mathscr{R}_{\bar{q}_{I}}, \widehat{\Phi}_{I}\right) .
\end{aligned}
$$

Let $I$ be the one-peak garland

$$
I=\mathscr{G}_{2}^{*}: \searrow_{2}^{1} \searrow_{4} \searrow_{4}
$$

The incidence matrix $C_{I}$, the Tits matrix $\widehat{C}_{I}$, and the CoxeterTits matrix $\widehat{C}_{I}=-\widehat{C}_{I} \cdot \widehat{C}_{I}^{-\operatorname{tr}}$ of $I$ are the following:

$$
\begin{gathered}
C_{I}=\left[\begin{array}{ccccc}
1 & 0 & 1 & 1 & 1 \\
0 & 1 & 1 & 1 & 1 \\
0 & 0 & 1 & 0 & 1 \\
0 & 0 & 0 & 1 & 1 \\
0 & 0 & 0 & 0 & 1
\end{array}\right], \\
\widehat{C}_{I}=\left[\begin{array}{cccc|c}
1 & 0 & 0 & 0 & -1 \\
0 & 1 & 0 & 0 & -1 \\
1 & 1 & 1 & 0 & -1 \\
1 & 1 & 0 & 1 & -1 \\
\hline 0 & 0 & 0 & 0 & 1
\end{array}\right], \\
\widehat{\operatorname{Cox}}_{I}=\left[\begin{array}{ccccc}
0 & 1 & 0 & 0 & 1 \\
1 & 0 & 0 & 0 & 1 \\
0 & 0 & 0 & 1 & 1 \\
0 & 0 & 1 & 0 & 1 \\
-1 & -1 & 1 & 1 & -1
\end{array}\right] .
\end{gathered}
$$

The Coxeter polynomial $\operatorname{cox}_{I}(t)$, the Tits quadratic form $\widehat{q}_{I}$ : $\mathbb{Z}^{5} \rightarrow \mathbb{Z}$, and the Coxeter-Tits transformation $\widehat{\Phi}_{I}: \mathbb{Z}^{5} \rightarrow$ $\mathbb{Z}^{5}$ of $I$ are

$$
\begin{gathered}
\operatorname{cox}_{I}(t)=t^{5}+t^{4}-2 t^{3}-2 t^{2}+t+1=F_{\mathbb{\mathbb { D }}_{4}}(t), \\
\widehat{q}_{I}(x)=x \cdot \widehat{C}_{I} \cdot x^{\operatorname{tr}}=x_{1}^{2}+x_{2}^{2}+x_{3}^{2}+x_{4}^{2}+x_{5}^{2} \\
+\left(x_{1}+x_{2}\right)\left(x_{3}+x_{4}\right)-\left(x_{1}+x_{2}+x_{3}+x_{4}\right) x_{5}, \\
\widehat{\Phi}_{I}(x)=x \cdot \widehat{\operatorname{Cox}}_{I}=\left(x_{2}-x_{5}, x_{1}-x_{5}, x_{4}+x_{5},\right. \\
\left.x_{3}+x_{5}, x_{1}+x_{2}+x_{3}+x_{4}-x_{5}\right),
\end{gathered}
$$

for $x=\left(x_{1}, x_{2}, x_{3}, x_{4}, x_{5}\right) \in \mathbb{Z}^{I} \equiv \mathbb{Z}^{5}$. Note that the $\widehat{\Phi}_{I^{-}}$ orbit $\mathcal{O}\left(e_{5}\right)$ of $e_{5}$ consists of two vectors $e_{5}$ and $\widehat{\Phi}_{I}\left(e_{5}\right)=$ $(-1,-1,1,1,-1)=\widehat{\Phi}_{I}^{-1}\left(e_{5}\right)$. Since

$$
\begin{aligned}
\widehat{q}_{I}(x)= & \left(x_{1}+\frac{1}{2} x_{3}+\frac{1}{2} x_{4}-\frac{1}{2} x_{5}\right)^{2} \\
& +\left(x_{2}+\frac{1}{2} x_{3}+\frac{1}{2} x_{4}-\frac{1}{2} x_{5}\right)^{2} \\
& +\frac{1}{2}\left(x_{3}-x_{4}\right)^{2}+\frac{1}{2} x_{5}^{2},
\end{aligned}
$$

then the form $\hat{q}_{I}: \mathbb{Z}^{5} \rightarrow \mathbb{Z}$ is positive semidefinite, is not positive definite,

$$
\operatorname{Ker} \widehat{q}_{I}=\mathbb{Z} \cdot \widehat{\mathbf{h}}_{I}, \quad \text { where } \widehat{\mathbf{h}}_{I}=(1,1,-1,-1,0) \text {, }
$$

and $\widehat{\Phi}_{I}\left(\widehat{\mathbf{h}}_{I}\right)=\widehat{\mathbf{h}}_{I}$. This means that $\widehat{q}_{I}$ is principal, but not $P$ critical; see [44]. One easily shows that the reduced Coxeter number of $I$ equals $\check{\mathbf{c}}_{I}=2$ and the Tits defect $\widehat{\partial}_{I}: \mathbb{Z}^{5} \rightarrow \mathbb{Z}$ of $I$ is given by $\widehat{\partial}_{I}(x)=-\left(x_{1}+x_{2}+x_{3}+x_{4}\right)$, because $\widehat{\Phi}_{I} \neq i d$ and $\widehat{\Phi}_{I}^{2}(v)=v+\widehat{\partial}_{I}(v) \cdot \widehat{\mathbf{h}}_{I}$, for any $v \in \mathbb{Z}^{5}$. The set $\mathscr{R}_{\widehat{q}_{I}}$ of roots of $\widehat{q}_{I}$ has the disjoint union decomposition (see (51))

$$
\mathscr{R}_{\widehat{q}_{I}}=\widehat{\partial}_{I}^{-} \mathscr{R}_{\widehat{q}_{I}} \cup \widehat{\partial}_{I}^{+} \mathscr{R}_{\widehat{q}_{I}} \cup \widehat{\partial}_{I}^{0} \mathscr{R}_{\widehat{q}_{I}},
$$

and $\widehat{\partial}_{I}^{-} \mathscr{R}_{\widehat{q}_{I}}, \widehat{\partial}_{I}^{+} \mathscr{R}_{\widehat{q}_{I}}, \widehat{\partial}_{I}^{0} \mathscr{R}_{\widehat{q}_{I}}$ are $\widehat{\Phi}_{I}$-invariant subsets of $\mathscr{R}_{\widehat{q}_{I}}$. Obviously, the $\widehat{\Phi}_{I}$-orbit $\mathscr{O}(v)$ of any $v \in \widehat{\partial}_{I}^{0} \mathscr{R}_{\widehat{q}_{I}}$ is of length two, whereas the $\widehat{\Phi}_{I}$-orbit $\mathcal{O}(w)$ of any vector $w \in \widehat{\partial}_{I}^{-} \mathscr{R}_{\widehat{q}_{I}} \cup$ $\widehat{\partial}_{I}^{+} \mathscr{R}_{\widehat{q}_{I}}$ is infinite. By (92), a vector $v=\left(v_{1}, v_{2}, v_{3}, v_{4}, v_{5}\right) \in \mathbb{Z}^{5}$ is a root of $\hat{q}_{I}: \mathbb{Z}^{5} \rightarrow \mathbb{Z}$ if and only if $\left(2 v_{1}+v_{3}+v_{4}-v_{5}\right)^{2}+$ $\left(2 v_{2}+v_{3}+v_{4}-v_{5}\right)^{2}+2\left(v_{3}-v_{4}\right)^{2}+2 v_{5}^{2}=4$. Hence, looking at all possible decompositions $4=a_{1}^{2}+a_{2}^{2}+2 a_{3}^{2}+2 a_{4}^{2}$, with $a_{1}, a_{2}, a_{3}, a_{4}, \in \mathbb{Z}$, we show that $v=\left(v_{1}, v_{2}, v_{3}, v_{4}, v_{5}\right) \in \mathbb{Z}^{5}$ is a root of $\hat{q}_{I}: \mathbb{Z}^{5} \rightarrow \mathbb{Z}$ if and only if $v$ or $\widehat{v}:=-v$ is one of the vectors listed in Table 1 or in Table 2.

(1) The $\widehat{\Phi}_{I}$-orbits in $\widehat{\mathscr{P}}_{I}:=\widehat{\partial}_{I}^{-} \mathscr{R}_{\widehat{q}_{I}}$. Since $\widehat{\partial}_{I}(u)<0$, if $u \in$ $\left\{e_{1}, e_{2}, e_{3}, e_{4}\right\}$ or $u$ is the vector $\mathbf{p}_{12}=(1,1,0,0,1)$, then the $\widehat{\Phi}_{I}$-orbits of the vectors $e_{1}, e_{2}, e_{3}, e_{4}, \mathbf{p}_{12}$ lie in $\widehat{\mathscr{P}}_{I}:=\widehat{\partial}_{I}^{-} \mathscr{R}_{\widehat{q}_{I}}$, because $\widehat{\mathscr{P}}_{I}$ is a $\widehat{\Phi}_{I}$-invariant subset of $\mathscr{R}_{\widehat{q}_{I}}$. It is easy to see that the $\widehat{\Phi}_{I}$-orbits consist of the vectors listed in Table 1 . 
TABLE 1

\begin{tabular}{|c|c|c|c|c|c|}
\hline$j$ & $\widehat{\Phi}_{I}^{j}\left(e_{1}\right)$ & $\widehat{\Phi}_{I}^{j}\left(e_{2}\right)$ & $\widehat{\Phi}_{I}^{j}\left(\mathbf{p}_{12}\right)$ & $\widehat{\Phi}_{I}^{j}\left(e_{3}\right)$ & $\widehat{\Phi}_{I}^{j}\left(e_{4}\right)$ \\
\hline : & $\vdots$ & $\vdots$ & $\vdots$ & $\vdots$ & : \\
\hline$j=7$ & $(-3,-2,3,3,1)$ & $(-2,-3,3,3,1)$ & $(-6,-6,7,7,1)$ & $(-3,-3,3,4,1)$ & $(-3,-3,4,3,1)$ \\
\hline$j=6$ & $(-2,-3,3,3,0)$ & $(-3,-2,3,3,0)$ & $(-5,-5,6,6,1)$ & $(-3,-3,4,3,0)$ & $(-3,-3,3,4,0)$ \\
\hline$j=5$ & $(-2,-1,2,2,1)$ & $(-1,-2,2,2,1)$ & $(-4,-4,5,5,1)$ & $(-2,-2,2,3,1)$ & $(-2,-2,3,2,1)$ \\
\hline$j=4$ & $(-1,-2,2,2,0)$ & $(-2,-1,2,2,0)$ & $(-3,-3,4,4,1)$ & $(-2,-2,3,2,0)$ & $(-2,-2,2,3,0)$ \\
\hline$j=3$ & $(-1,0,1,1,1)$ & $(0,-1,1,1,1)$ & $(-2,-2,3,3,1)$ & $(-1,-1,1,2,1)$ & $(-1,-1,2,1,1)$ \\
\hline$j=2$ & $(0,-1,1,1,0)$ & $(-1,0,1,1,0)$ & $(-1,-1,2,2,1)$ & $(-1,-1,2,1,0)$ & $(-1,-1,1,2,0)$ \\
\hline$j=1$ & $(0,1,0,1,0)$ & $(1,0,0,0,1)$ & $(0,0,1,1,1)$ & $(0,0,0,1,1)$ & $(0,0,1,0,1)$ \\
\hline$j=0$ & $(1,0,0,0,0)$ & $(0,1,0,0,0)$ & $(1,1,0,0,1)$ & $(0,0,1,0,0)$ & $(0,0,0,1,0)$ \\
\hline$j=-1$ & $(1,2,-1,-1,1)$ & $(2,1,-1,-1,1)$ & $(2,2,-1,-1,1)$ & $(1,1,-1,0,1)$ & $(1,1,0,-1,1)$ \\
\hline$j=-2$ & $(2,1,-1,-1,0)$ & $(1,2,-1,-1,0)$ & $(3,3,-2,-2,1)$ & $(1,1,0,-1,0)$ & $(1,1,-1,0,0)$ \\
\hline$j=-3$ & $(2,3,-2,-2,1)$ & $(3,2,-2,-2,1)$ & $(4,4,-3,-3,1)$ & $(2,2,-2,-1,1)$ & $(2,2,-1,-2,1)$ \\
\hline$j=-4$ & $(3,2,-2,-2,0)$ & $(2,3,-2,-2,0)$ & $(5,5,-4,-4,1)$ & $(2,2,-1,-2,0)$ & $(2,2,-2,-1,0)$ \\
\hline$j=-5$ & $(3,4,-3,-3,1)$ & $(4,3,-3,-3,1)$ & $(6,6,-5,-5,1)$ & $(3,3,-3,-2,1)$ & $(3,3,-2,-3,1)$ \\
\hline$j=-6$ & $(4,3,-3,-3,0)$ & $(3,4,-3,-3,0)$ & $(7,7,-6,-6,1)$ & $(3,3,-2,-3,0)$ & $(3,3,-3,-2,0)$ \\
\hline$j=-7$ & $(4,5,-4,-4,1)$ & $(5,4,-4,-4,1)$ & $(8,8,-7,-7,1)$ & $(4,4,-4,-3,1)$ & $(4,4,-3,-4,1)$ \\
\hline$\vdots$ & $\vdots$ & $\vdots$ & $\vdots$ & $\vdots$ & $\vdots$ \\
\hline
\end{tabular}

TABLE 2

\begin{tabular}{|c|c|c|c|c|c|c|}
\hline$j$ & $u^{(j)}$ & $u_{+}^{(j)}$ & $w^{(j)}$ & $w_{+}^{(j)}$ & $v^{(j)}$ & $v_{+}^{(j)}$ \\
\hline$j=0$ & $-e_{5}=(0,0,0,0,-1)$ & $(1,1,-1,-1,1)$ & $(1,0,0,-1,0)$ & $(0,1,-1,0,0)$ & $(0,1,0,-1,0)$ & $(1,0,-1,0,0)$, \\
\hline$j=1$ & $(2,2,-2,-2,1)$ & $(1,1,-1,-1,-1)$ & $(1,2,-2,-1,0)$ & $(2,1,-1,-2,0)$ & $(2,1,-2,-1,0)$ & $(1,2,-1,-2,0)$ \\
\hline$j=2$ & $(2,2,-2,-2,-1)$ & $(3,3,-3,-3,1)$ & $(3,2,-2,-3,0)$ & $(2,3,-3,-2,0)$ & $(2,3,-2,-3,0)$ & $(3,2,-3,-2,0)$ \\
\hline$j=3$ & $(4,4,-4,-4,1)$ & $(3,3,-3,-3,-1)$ & $(3,4,-4,-3,0)$ & $(4,3,-3,-4,0)$ & $(4,3,-4,-3,0)$ & $(3,4,-3,-4,0)$ \\
\hline$j=4$ & $(4,4,-4,-4,-1)$ & $(5,5,-5,-5,1)$ & $(5,4,-4,-5,0)$ & $(4,5,-5,-4,0)$ & $(4,5,-4,-5,0)$ & $(5,4,-5,-4,0)$ \\
\hline$j=5$ & $(6,6,-6,-6,1)$ & $(5,5,-5,-5,-1)$ & $(5,6,-6,-5,0)$ & $(6,5,-5,-6,0)$ & $(6,5,-6,-5,0)$ & $(5,6,-5,-6,0)$ \\
\hline$j=6$ & $(6,6,-6,-6,-1)$ & $(7,7,-7,-7,1)$ & $(7,6,-6,-7,0)$ & $(6,7,-7,-6,0)$ & $(6,7,-6,-7,0)$ & $(7,6,-7,-6,0)$ \\
\hline$j=7$ & $(8,8,-8,-8,1)$ & $(7,7,-7,-7,-1)$ & $(7,8,-8,-7,0)$ & $(8,7,-7,-8,0)$ & $(8,7,-8,-7,0)$ & $(7,8,-7,-8,0)$ \\
\hline$\vdots$ & $\vdots$ & $\vdots$ & $\vdots$ & $\vdots$ & $\vdots$ & $\vdots$ \\
\hline
\end{tabular}

Throughout this section, we freely use the $\widehat{\Phi}_{I}$-mesh terminology and notation introduced in $[2,34,40]$.

(2) $\widehat{\Phi}_{I}$-mesh quiver $\Gamma\left(\widehat{\mathscr{P}}_{I}, \widehat{\Phi}_{I}\right)=\Gamma\left(\widehat{\partial}_{I}^{-} \mathscr{R}_{\widehat{q}_{I}}, \widehat{\Phi}_{I}\right)$. It follows from our earlier remarks that the set $\widehat{\mathscr{P}}_{I}:=\widehat{\partial}_{I}^{-} \mathscr{R}_{\widehat{q}_{I}}$ of the negative defect roots of $\widehat{q}_{I}$ splits into the five $\widehat{\Phi}_{I}$-orbits $\mathcal{O}\left(e_{1}\right)$, $\mathcal{O}\left(e_{2}\right), \mathcal{O}\left(e_{3}\right), \mathcal{O}\left(e_{4}\right), \mathcal{O}\left(\mathbf{p}_{12}\right)$. By applying the mesh toroidal algorithm defined in $[2,34]$, one constructs the following infinite $\widehat{\Phi}_{I}$-mesh translation quiver of the negative defect roots of $\widehat{q}_{I}$; see Figure 2 , where we set $\widehat{a}:=-a$ for any positive integer $a \geq 1$.

(3) $\widehat{\Phi}_{I}$-mesh quiver $\Gamma\left(\widehat{Q}_{I}, \widehat{\Phi}_{I}\right)=\Gamma\left(\widehat{\partial}_{I}^{+} \mathscr{R}_{\widehat{q}_{I}}, \widehat{\Phi}_{I}\right)$. Since the group isomorphism $\mathbb{Z}^{I} \rightarrow \mathbb{Z}^{I}, v \mapsto-v$, carries roots to roots, $\widehat{\Phi}_{I^{-}}$-meshes to $\widehat{\Phi}_{I^{-}}$-meshes, and $\widehat{\Phi}_{I^{-}}$orbits to $\widehat{\Phi}_{I^{-}}$ orbits, then it defines the bijections $\widehat{\partial}_{I}^{-} \mathscr{R}_{\widehat{q}_{I}} \rightarrow \widehat{\partial}_{I}^{+} \mathscr{R}_{\widehat{q}_{I}}$ and $\widehat{\partial}_{I}^{0} \mathscr{R}_{\widehat{q}_{I}} \rightarrow \widehat{\partial}_{I}^{0} \mathscr{R}_{\widehat{q}_{I}}$, because $\widehat{\partial}_{I}(-v)=-\widehat{\partial}_{I}(v)$. It follows that the set $\widehat{Q}_{I}:=\widehat{\partial}_{I}^{+} \mathscr{R}_{\widehat{q}_{I}}$ of the positive defect roots of $\widehat{q}_{I}$ splits into the five $\widehat{\Phi}_{I}$-orbits $\mathcal{O}\left(\widehat{e}_{1}\right), \mathcal{O}\left(\widehat{e}_{2}\right), \mathcal{O}\left(\widehat{e}_{3}\right), \mathcal{O}\left(\widehat{e}_{4}\right), \mathcal{O}\left(\widehat{\mathbf{p}}_{12}\right)$, and one constructs the infinite $\widehat{\Phi}_{I}$-mesh translation quiver

$$
\Gamma\left(\widehat{Q}_{I}, \widehat{\Phi}_{I}\right)=\Gamma\left(\widehat{\partial}_{I}^{+} \mathscr{R}_{\widehat{q}_{I}}, \widehat{\Phi}_{I}\right)
$$

of the positive defect roots of $\hat{q}_{I}$ by interchanging any vector $v$ in $\Gamma\left(\widehat{\mathscr{P}}_{I}, \widehat{\Phi}_{I}\right)=\Gamma\left(\widehat{\partial}_{I}^{-} \mathscr{R}_{\widehat{q}_{I}}, \widehat{\Phi}_{I}\right)$ with its negative $\widehat{v}:=-v$. 
(4) $\widehat{\Phi}_{I}$-mesh quiver $\Gamma\left(\widehat{\partial}_{I}^{0} \mathscr{R}_{\widehat{q}_{I}}, \widehat{\Phi}_{I}\right)$. By the equality $\widehat{\Phi}_{I}^{2}(v)=v-\widehat{\partial}_{I}(v) \cdot \widehat{\mathbf{h}}_{I}$, the $\widehat{\Phi}_{I}$-orbit of any $v \in \widehat{\partial}_{I}^{0} \mathscr{R}_{\widehat{q}_{I}}$ consists of two vectors $v$ and $\widehat{\Phi}_{I}(v)$. Now, we show that the $\widehat{\Phi}_{I}$-orbits in $\widehat{\partial}_{I}^{0} \mathscr{R}_{\widehat{q}_{I}}$ form a $\widehat{\Phi}_{I}$-mesh translation quiver $\Gamma\left(\widehat{\partial}_{I}^{0} \mathscr{R}_{\widehat{q}_{I}}, \widehat{\Phi}_{I}\right)$.

Note that $\widehat{\Phi}_{I}\left(e_{5}\right)=(-1,-1,1,1,-1), \widehat{\Phi}_{I}^{2}\left(e_{5}\right)=e_{5}, \widehat{\partial}_{I}\left(e_{5}\right)=$ 0 , and $\widehat{\partial}_{I}\left(\widehat{\Phi}_{I}\left(e_{5}\right)\right)=0$. It follows that the two-element $\widehat{\Phi}_{I^{-}}$ orbits of $e_{5}$ and $-e_{5}$ lie in $\widehat{\partial}_{I}^{0} \mathscr{R}_{\widehat{q}_{I}}$. Moreover, the vectors

$$
\begin{gathered}
u_{+}^{(1)}=(1,1,-1,-1,-1), \quad-u_{+}^{(1)}, \\
w^{(0)}=(1,0,0,-1,0), \\
w_{+}^{(0)}=\widehat{\Phi}_{I}\left(w^{(0)}\right)=(0,1,-1,0,0), \\
v^{(0)}=(0,1,0,-1,0), \\
v_{+}^{(0)}=\widehat{\Phi}_{I}\left(v^{(0)}\right)=(1,0,-1,0,0) \\
w_{+}^{(1)}=(2,1,-1,-2,0), \\
v_{+}^{(1)}=(1,2,-1,-2,0)
\end{gathered}
$$

belong to $\widehat{\partial}_{I}^{0} \mathscr{R}_{\widehat{q}_{I}}$. It is easy to see that we have the following $\widehat{\Phi}_{I}^{2}$-mesh quivers of vectors in $\widehat{\partial}_{I}^{0} \mathscr{R}_{\widehat{q}_{I}}$ :
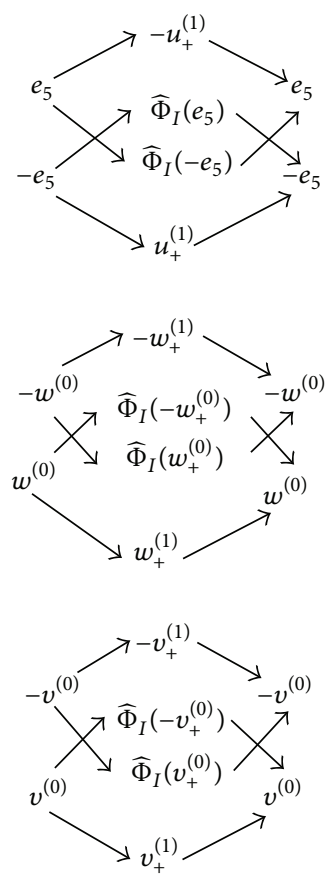

Note that the $\widehat{\Phi}_{I}$-orbit of $u_{+}^{(1)}$ consists of the following two vectors:

$$
u^{(1)}=(2,-2,-2,-2,1), \quad u_{+}^{(1)}=\widehat{\Phi}_{I}\left(u^{(1)}\right) .
$$

By (92), a vector $v=\left(v_{1}, v_{2}, v_{3}, v_{4}, v_{5}\right) \in \mathbb{Z}^{5}$ is a root of $\widehat{q}_{I}$ : $\mathbb{Z}^{5} \rightarrow \mathbb{Z}$ of defect zero if and only if

$$
\begin{gathered}
\left(2 v_{1}+v_{3}+v_{4}-v_{5}\right)^{2}+\left(2 v_{2}+v_{3}+v_{4}-v_{5}\right)^{2} \\
+2\left(v_{3}-v_{4}\right)^{2}+2 v_{5}^{2}=4 \\
v_{1}+v_{2}+v_{3}+v_{4}=0 .
\end{gathered}
$$

It follows that $v$ or $-v$ belongs to any of the six series of roots presented in Table 2.

Hence, we conclude that the $\widehat{\Phi}_{I}$-orbits in the set $\widehat{\partial}_{I}^{0} \mathscr{R}_{\widehat{q}_{I}}$ form three $\widehat{\Phi}_{I}$-mesh quivers $\mathscr{T}_{u}, \mathscr{T}_{w}, \mathscr{T}_{v}$, and each of them has the form of infinite two-surface tube of rank 2 :

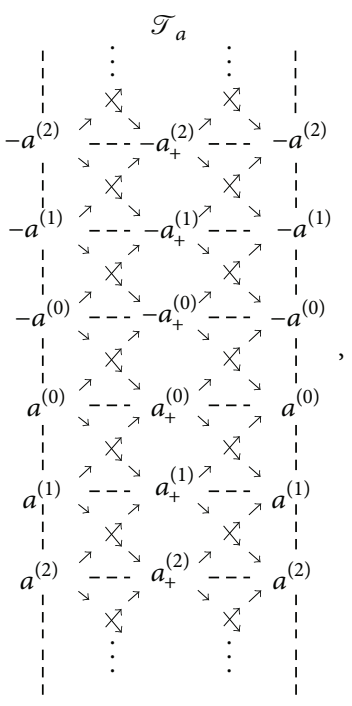

where $a$ is one of the vectors

$$
\begin{aligned}
& u=u^{(0)}=(0,0,0,0,-1), \\
& w=w^{(0)}=(1,0,0,-1,0), \\
& v=v^{(0)}=(0,1,0,-1,0) .
\end{aligned}
$$

(5) $\widehat{\Phi}_{I}$-mesh quiver $\Gamma\left(\widehat{\partial}_{I}^{0} \mathscr{R}_{\widehat{q}_{I}} \cup \operatorname{Ker} \widehat{q}_{I}, \widehat{\Phi}_{I}\right)$. We recall that $\operatorname{Ker} \widehat{q}_{I}=\mathbb{Z} \cdot \widehat{\mathbf{h}}_{I}$, where $\widehat{\mathbf{h}}_{I}=(1,1,-1,-1,0)$. Note that

$$
\widehat{\Phi}_{I}\left(\widehat{\mathbf{h}}_{I}\right)=\widehat{\mathbf{h}}_{I}, \quad \widehat{\Phi}_{I}\left(m \cdot \widehat{\mathbf{h}}_{I}\right)=m \cdot \widehat{\mathbf{h}}_{I}, \text { for any } m \in \mathbb{Z} .
$$

Obviously, the vectors lying in $\operatorname{Ker} \widehat{q}_{I}$ form the $\widehat{\Phi}$-mesh translation quiver $\widehat{\mathscr{T}}_{\widehat{\mathbf{h}}_{I}}$ presented in (108).

Now, we construct from the $\widehat{\Phi}_{I}$-orbits in the set $\partial_{I}^{0} \mathscr{R}_{\widehat{q}_{I}} U$ $\operatorname{Ker} \widehat{q}_{I}$ an infinite $\widehat{\Phi}_{I}$-mesh translation quiver. For this purpose, we note that the following six vectors

$$
-e_{5}, e_{5}, \widehat{\Phi}_{I}\left(-e_{5}\right), \widehat{\Phi}_{I}\left(e_{5}\right), \quad-\widehat{\mathbf{h}}_{I}, \widehat{\mathbf{h}}_{I}
$$

form two $\widehat{\Phi}_{I}$-meshes of width 1 . If we complete them by the three vectors

$$
0, u_{+}^{(1)}:=(1,1,-1,-1,-1), \quad-u_{+}^{(1)},
$$




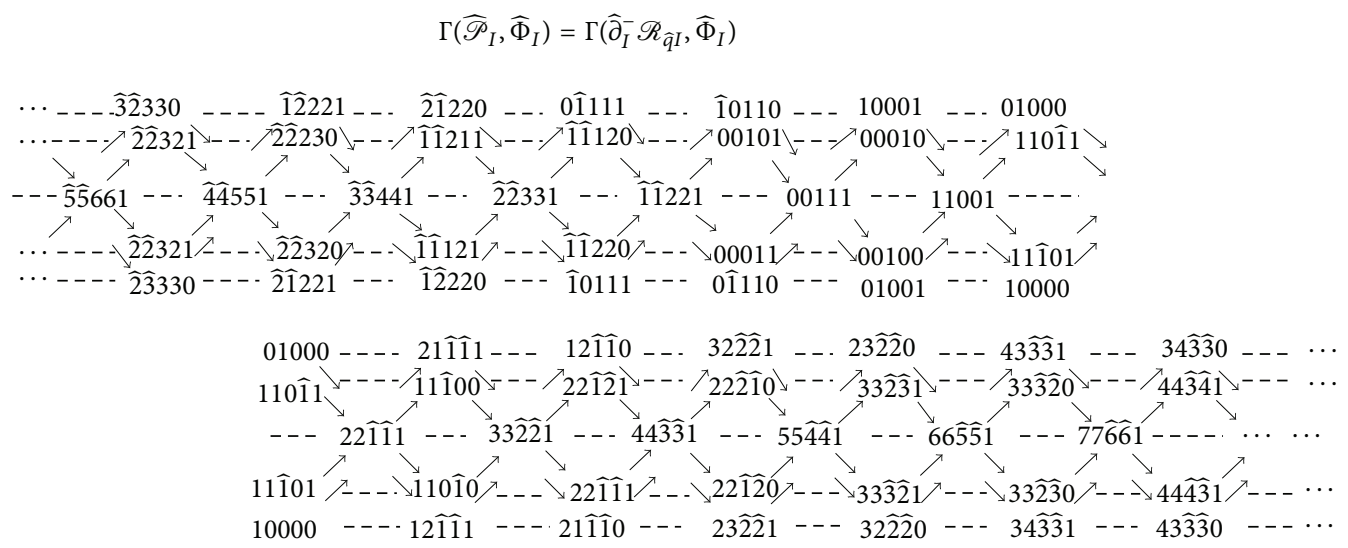

Figure 2

we get the $\widehat{\Phi}_{I}$-mesh quiver

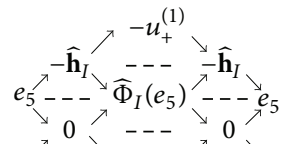

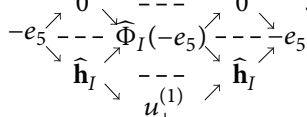

Analogously, we construct the following two $\widehat{\Phi}_{I}$-mesh quivers

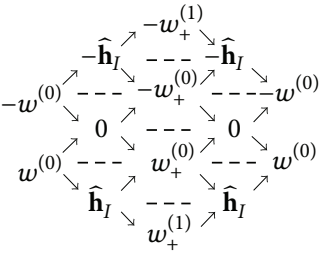

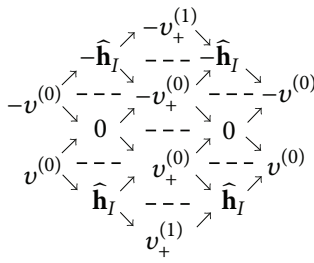

where

$$
\begin{gathered}
u^{(1)}=(2,-2,-2,-2,1), \\
u_{+}^{(1)}=\widehat{\Phi}_{I}\left(u^{(1)}\right), \\
w^{(0)}=(1,0,0,-1,0), \\
w_{+}^{(0)}=\widehat{\Phi}_{I}\left(w^{(0)}\right)=(0,1,-1,0,0),
\end{gathered}
$$

$$
\begin{gathered}
v^{(0)}=(0,1,0,-1,0), \\
v_{+}^{(0)}=\widehat{\Phi}_{I}\left(v^{(0)}\right)=(1,0,-1,0,0), \\
w_{+}^{(1)}=(2,1,-1,-2,0), \\
v_{+}^{(1)}=(1,2,-1,-2,0) .
\end{gathered}
$$

We recall that if $v \in \widehat{\partial}_{I}^{0} \mathscr{R}_{\widehat{q}_{I}}$, then $v$ or $-v$ is one of the vectors presented in Table 2 . It follows that the $\widehat{\Phi}_{I}$-orbits in $\widehat{\partial}_{I}^{0} \mathscr{R}_{\widehat{q}_{I}} \cup$ $\mathbb{Z} \cdot \widehat{\mathbf{h}}_{I}$ form three infinite $\widehat{\Phi}_{I}$-mesh sand-glass tubes $\widehat{\mathscr{T}}_{u}, \widehat{\mathscr{T}}_{w}$, $\widehat{\mathscr{T}}_{v}$ of rank $(2,1)$, and each of them has the shape presented in (109) 


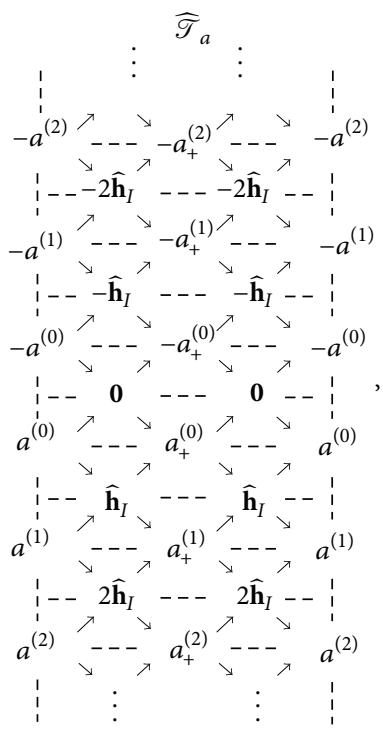

where $a$ is one of the vectors

$$
\begin{aligned}
& u=u^{(0)}=(0,0,0,0,-1), \\
& w=w^{(0)}=(1,0,0,-1,0), \\
& v=v^{(0)}=(0,1,0,-1,0)
\end{aligned}
$$

Construct the disjoint union $\widehat{\mathscr{T}}_{u} \cup \widehat{\mathscr{T}}_{w} \cup \widehat{\mathscr{T}}_{v}$ of the tubes $\widehat{\mathscr{T}}_{u}$, $\widehat{\mathscr{T}}_{w}, \widehat{\mathscr{T}}_{v}$, and note that each of them contains the tube $\widehat{\mathscr{T}}_{\widehat{\mathbf{h}}_{I}}$. By making the identification of the vectors $m \cdot \widehat{\mathbf{h}}_{I}$, with $m \in \mathbb{Z}$, lying in the corresponding $\widehat{\Phi}_{I^{-}}$-orbits, we get the quotient $\widehat{\Phi}_{I^{-}}$ mesh translation quiver

$$
\Gamma\left(\widehat{\partial}_{I}^{0} \mathscr{R}_{\widehat{q}_{I}} \cup \operatorname{Ker} \widehat{q}_{I}, \widehat{\Phi}_{I}\right)=\frac{\widehat{\mathscr{T}}_{u} \cup \widehat{\mathscr{T}}_{w} \cup \widehat{\mathscr{T}}_{v}}{\simeq}
$$

that has a shape of a threefold sand-glass tube of rank $(2,2,2,1)$ in the sense of $[40]$. It is obtained from the disjoint union of three copies of the onefold sand-glass tube of rank $(2,1)$ presented in Figure 3 (see also [34, Figure 5.8]) by making an obvious identification of their waist vectors.

(6) $A \mathbb{Z}$-congruence of the bigraph $\widehat{\Delta}_{I}$ with the Euclidean diagram $\widetilde{\mathbb{D}}_{4}$. Since we have $\operatorname{cox}_{I}(t)=F_{\widetilde{\mathbb{D}}_{4}}(t)=t^{5}+t^{4}-2 t^{3}-$ $2 t^{2}+t+1$ and $\operatorname{specc}_{I}=\operatorname{specc}_{\widetilde{\mathbb{D}}_{4}}$, the Euclidean diagram $\widetilde{\mathbb{D}}_{4}$ is the diagram $D I$ associated to $I$. A technique developed in [2,
$17,18,34,40]$ allows us to construct a $\mathbb{Z}$-invertible matrix $B \in$ $\mathrm{Gl}(5, \mathbb{Z})$ such that the following diagrams are commutative:

$$
\begin{gathered}
\mathbb{Z}^{5} \times \mathbb{Z}^{5} \stackrel{\widehat{b}_{I}}{\longrightarrow} \mathbb{Z} \\
h_{B} \times h_{B} \uparrow \cong b_{\widetilde{\mathbb{D}}_{4}} \\
\mathbb{Z}^{5} \times \mathbb{Z}^{5} \\
\mathbb{Z}^{5} \stackrel{\widehat{q}_{I}}{\longrightarrow} \mathbb{Z} \\
h_{B} \uparrow \nearrow^{q_{\mathbb{D}_{4}}} \\
\mathbb{Z}^{5}
\end{gathered}
$$

where $b_{\widetilde{\mathbb{D}}_{4}}$ and $q_{\widetilde{\mathbb{D}}_{4}}$ are the forms of the Euclidean diagram

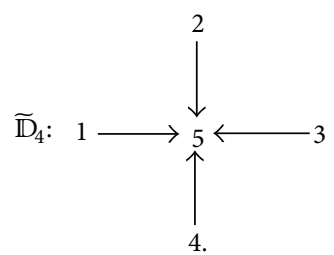

defined by the formulae $b_{\widetilde{\mathbb{D}}_{4}}(x, y)=x_{1} y_{1}+x_{2} y_{2}+x_{3} y_{3}+x_{4} y_{4}+$ $x_{5} y_{5}-\left(x_{1}+x_{2}+x_{3}+x_{4}\right) y_{5}=x \cdot \check{G}_{\widetilde{\mathbb{D}}_{4}} \cdot y^{\operatorname{tr}}, q_{\widetilde{\mathbb{D}}_{4}}(x)=b_{\widetilde{\mathbb{D}}_{4}}(x, x)$, for $x, y \in \mathbb{Z}^{5}, h_{B}: \mathbb{Z}^{5} \rightarrow \mathbb{Z}^{5}$ is the group automorphism defined by the formula $h_{B}(x)=x \cdot B$, and

$$
\begin{aligned}
& B=\left[\begin{array}{ccccc}
1 & 0 & -1 & -1 & 0 \\
0 & 0 & -1 & 0 & -1 \\
0 & 0 & 0 & -1 & -1 \\
0 & 1 & -1 & -1 & 0 \\
0 & 0 & 1 & 1 & 1
\end{array}\right], \\
& \check{G}_{\widetilde{\mathbb{D}}_{4}}=\left[\begin{array}{ccccc}
1 & 0 & 0 & 0 & -1 \\
0 & 1 & 0 & 0 & -1 \\
0 & 0 & 1 & 0 & -1 \\
0 & 0 & 0 & 1 & -1 \\
0 & 0 & 0 & 0 & 1
\end{array}\right] .
\end{aligned}
$$

It is easy to check that the equality $\check{G}_{\widetilde{\mathbb{D}}_{4}}=B \cdot \widehat{C}_{I} \cdot B^{\operatorname{tr}}$ holds, and therefore the diagrams (112) are commutative. Furthermore, by the same technique, we construct another matrix

$$
B_{1}=\left[\begin{array}{ccccc}
-1 & 0 & 0 & 0 & -1 \\
0 & 0 & 0 & -1 & 0 \\
0 & 0 & -1 & 0 & 0 \\
0 & -1 & 0 & 0 & -1 \\
1 & 1 & 0 & 0 & 1
\end{array}\right]
$$

such that the equality $\check{G}_{\widetilde{\mathbb{D}}_{4}}=B_{1} \cdot \widehat{C}_{I} \cdot B_{1}^{\text {tr }}$ holds.

\section{Concluding Remarks}

6.1 It follows from Lemma 3 and the results obtained recently in $[3,4]$ that for any connected positive (resp., 


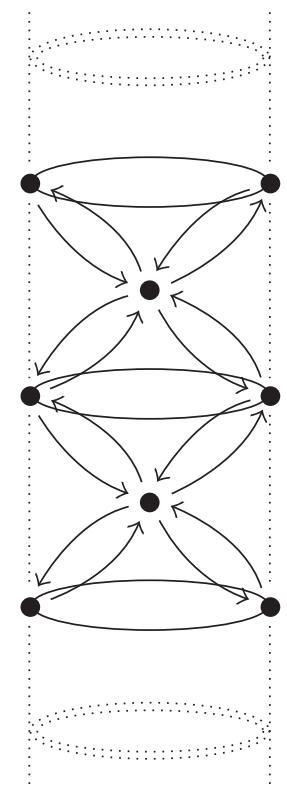

FIgURE 3: Sand-glass tube of rank $(2,1)$.

principal) poset $J$, there exists a simply laced Dynkin diagram $D J \in\left\{\mathbb{A}_{m}, \mathbb{D}_{m}, \mathbb{E}_{6}, \mathbb{E}_{7}, \mathbb{E}_{8}\right\}$ (resp., a simply laced Euclidean diagram $D J$ ), uniquely determined by $J$, such that the symmetric Gram matrices $G_{J}, G_{D J}$ are $\mathbb{Z}$-congruent.

Analogous Coxeter spectral classification of one-peak posets $I$, with almost $P$-critical Tits form $\widehat{q}_{I}: \mathbb{Z}^{I} \rightarrow$ $\mathbb{Z}$, is obtained in [33] by a reduction to computer calculations.

6.2. Although the Coxeter spectral classification problem for arbitrary finite posets remains unsolved, we have a solution for positive one-peak posets. Indeed, it follows from the results in [17] that for any onepeak positive poset $J$, there exists a simply laced Dynkin diagram $D J \in\left\{\mathbb{A}_{s}, \mathbb{D}_{n}, \mathbb{E}_{6}, \mathbb{E}_{7}, \mathbb{E}_{8}\right\}$ (uniquely determined by $J$ ) such that $\operatorname{specc}_{J}=\operatorname{specc}_{D J}$, the nonsymmetric Gram matrices $\check{G}_{J}, \check{G}_{D J}$ are $\mathbb{Z}$ congruent, and the symmetric Gram matrices $G_{J}, G_{D J}$ are $\mathbb{Z}$-congruent.

6.3. We can determine the diagram $D J$ as follows. Fix an upper-triangular numbering $\left\{a_{1}, \ldots, a_{m}\right\}$ of elements of $J$. Then, the incidence matrix $C_{J} \in \mathbb{M}_{m}(\mathbb{Z})$ is uppertriangular, and the Euler matrix $\bar{C}_{J}:=C_{J}^{-1}$ is also upper triangular. Then, the Euler edge-bipartite graph $\bar{\Delta}_{J}$ (33) is loop-free, and we have $C_{\bar{\Delta}_{J}}=\bar{C}_{J}$. Hence, the symmetric Gram matrices $G_{\bar{\Delta}_{J}}, \bar{G}_{J}$ coincide, and, by Lemma 3, the poset $J$ is positive (resp., principal) if and only if the bigraph $\Delta_{J}$ is positive (resp., principal). By applying to $\bar{\Delta}_{J}$ the inflation algorithm constructed in $[4,21]$ (see also [45]), we get (in a finite number of steps) an edge-bipartite graph $D \Delta_{J}$ such that the symmetric Gram matrix $G_{\bar{\Delta}_{I}}=\bar{G}_{J}$ is $\mathbb{Z}$-congruent with the symmetric Gram matrix $G_{D \Delta_{J}}$, and the edgebipartite graph $D \Delta_{J}$ has no dotted edges; that is, $D \Delta_{J}$ is a (multi) graph. We set $D J:=D \Delta_{J}$. It follows from the results in $[3,4]$ that $D J$ is a simply laced Dynkin diagram, if $J$ is positive, and $D J$ is a simply laced Euclidean diagram, if $J$ is principal. Moreover, the matrix $\bar{G}_{J}$ is $\mathbb{Z}$-congruent with $G_{D J}$. Since the incidence Gram matrix $G_{J}$ of $J$ is $\mathbb{Z}$-congruent with the matrix $\bar{G}_{J}$ (by Proposition 5), then the matrices $G_{J}$ and $G_{D J}$ are $\mathbb{Z}$-congruent.

6.4. Although we can apply in 6.3 the inflation algorithm to the incidence edge-bipartite graph $\Delta_{J}$, we use in the construction of $D J$ the Euler edge-bipartite graph $\bar{\Delta}_{J}$, because the number of nonzero entries in the Euler matrix $\bar{C}_{J}:=C_{J}^{-1}$ does not increase the number for the matrix $C_{J}$; see [28, Proposition 2.12]. It follows that the number of the dotted edges in $\bar{\Delta}_{J}$ does not increase the number of the dotted edges in $\Delta_{J}$, and the use in 6.3 the bigraph $\bar{\Delta}_{J}$ reduces the time of calculation in the procedure $\bar{\Delta}_{J} \mapsto D J$.

\section{Acknowledgment}

The research is supported by Polish Research Grant NCN 2011/03/B/ST1/00824.

\section{References}

[1] D. Simson, "Integral bilinear forms, Coxeter transformations and Coxeter polynomials of finite posets," Linear Algebra and Its Applications, vol. 433, no. 4, pp. 699-717, 2010.

[2] D. Simson, "Algorithms determining matrix morsifications, Weyl orbits, Coxeter polynomials and mesh geometries of roots for Dynkin diagrams," Fundamenta Informatica, vol. 123, 2013. 
[3] D. Simson, "A framework for Coxeter spectral analysis of loopfree edge-bipartite graphs, their rational morsifications and mesh geometries of root orbits," Fundamenta Informaticae, vol. 125, 2013.

[4] D. Simson, "A Coxeter-Gram classification of simply-laced edge bipartite graphs," SIAM Journal on Discrete Mathematics, vol. 27, 2013.

[5] T. Zaslavsky, "Signed graphs," Discrete Applied Mathematics, vol. 4, no. 1, pp. 47-74, 1982.

[6] G. R. Belitskii and V. V. Sergeichuk, "Complexity of matrix problems," Linear Algebra and its Applications, vol. 361, pp. 203222, 2003.

[7] V. M. Bondarenko, "Representations of bundles of semichained sets and their applications," Algebra i Analiz, vol. 3, no. 5, pp. 38-61, 1991 (Russian).

[8] V. M. Bondarenko, "Representations of bundles of semichained sets and their applications," St. Petersburg Mathematical Journal, vol. 3, pp. 973-996, 1992.

[9] V. M. Bondarenko, "Minimax isomorphism algorithm and primitive posets," Algebra and Discrete Mathematics, vol. 12, no. 2, pp. 31-37, 2011.

[10] V. M. Bondarenko, T. G. Gerasimova, and V. V. Sergeichuk, "Pairs of mutually annihilating operators," Linear Algebra and its Applications, vol. 430, no. 1, pp. 86-105, 2009.

[11] V. M. Bondarenko and M. V. Styopochkina, "On posets of width two with positive Tits form," Algebra and Discrete Mathematics, vol. 2, no. 2, pp. 20-35, 2005.

[12] V. M. Bondarenko and M. V. Styopochkina, "On finite posets of P-finite type and their Tits forms," Algebra and Discrete Mathematics, vol. 2, pp. 17-21, 2006.

[13] V. M. Bondarenko and M. V. Stepochkina, "(Min, max)equivalence of posets, and nonnegative Tits forms," Ukrains'kyi Matematychnyi Zhurnal, vol. 60, no. 9, pp. 1157-1167, 2008.

[14] V. M. Bondarenko and M. V. Stepochkina, "Description of partially ordered sets that are critical with respect to the nonnegativity of the quadratic Tits form," Ukrains'kyi Matematychnyi Zhurnal, vol. 61, no. 5, pp. 611-624, 2009.

[15] R. A. Brualdi, "Spectra of digraphs," Linear Algebra and its Applications, vol. 432, no. 9, pp. 2181-2213, 2010.

[16] P. Gabriel and A. V. Roiter, Representations of Finite Dimensional Algebras, Algebra VIII, vol. 73 of Encyclopaedia of Mathematical Sciences, Springer, Berlin, Germany, 1992.

[17] M. Gạsiorek and D. Simson, "One-peak posets with positive quadratic Tits form, their mesh translation quivers of roots, and programming in Maple and Python," Linear Algebra and its Applications, vol. 436, no. 7, pp. 2240-2272, 2012.

[18] M. Gąsiorek and D. Simson, "A computation of positive onepeak posets that are Tits-sincere," Colloquium Mathematicum, vol. 127, pp. 83-103, 2012.

[19] A. Kisielewicz and M. Szykuła, "Rainbow induced subgraphs in proper vertex colorings," Fundamenta Informaticae, vol. 111, no. 4, pp. 437-451, 2011.

[20] J. Kosakowska, "A classification of two-peak sincere posets of finite prinjective type and their sincere prinjective representations," Colloquium Mathematicum, vol. 87, no. 1, pp. 27-77, 2001.

[21] J. Kosakowska, "Inflation algorithms for positive and principal edge-bipartite graphs and unit quadratic forms," Fundamenta Informaticae, vol. 119, pp. 149-162, 2012.

[22] S. Ladkani, "On the periodicity of Coxeter transformations and the non-negativity of their Euler forms," Linear Algebra and its Applications, vol. 428, no. 4, pp. 742-753, 2008.
[23] P. Lakatos, "Additive functions on trees," Colloquium Mathematicum, vol. 89, no. 1, pp. 135-145, 2001.

[24] S. A. Ovsienko, "Integral weakly positive forms," in Schur Matrix Problems and Quadratic Forms, pp. 3-17, Akademiia Nauk SSSR, 1978.

[25] V. V. Sergeichuk, "Canonical matrices for linear matrix problems," Linear Algebra and its Applications, vol. 317, no. 1-3, pp. 53-102, 2000.

[26] D. Simson, Linear Representations of Partially Ordered Sets and Vector Space Categories, Algebra, Logic and Applications, vol. 4, Gordon and Breach Science, New York, NY, USA, 1992.

[27] D. Simson, "Posets of finite prinjective type and a class of orders," Journal of Pure and Applied Algebra, vol. 90, no. 1, pp. 77-103, 1993.

[28] D. Simson, "Incidence coalgebras of intervally finite posets, their integral quadratic forms and comodule categories," Colloquium Mathematicum, vol. 115, no. 2, pp. 259-295, 2009.

[29] A. P. Wojda, "A condition for a graph to contain $k$-matching," Discrete Mathematics, vol. 276, no. 1-3, pp. 375-378, 2004.

[30] A. P. Wojda, M. Woźniak, and I. A. Zioło, "On selfcomplementary cyclic packing of forests," Electronic Journal of Combinatorics, vol. 14, no. 1, 11 pages, 2007.

[31] Y. B. Zhang, "Eigenvalues of Coxeter transformations and the structure of regular components of an Auslander-Reiten quiver," Communications in Algebra, vol. 17, no. 10, pp. 2347-2362, 1989.

[32] Ju. A. Drozd, "Coxeter transformations and representations of partially ordered sets," Functional Analysis and its Applications, vol. 8, no. 3, pp. 219-225, 1974.

[33] A. Polak and D. Simson, "One-peak posets with almost $P$ critical Tits form and a spectral Coxeter classification using computer algebra tools," European Journal of Combinatorics. To appear.

[34] D. Simson, "Mesh algorithms for solving principal Diophantine equations, sand-glass tubes and tori of roots," Fundamenta Informaticae, vol. 109, no. 4, pp. 425-462, 2011.

[35] R. A. Horn and V. V. Sergeichuk, "Congruences of a square matrix and its transpose," Linear Algebra and its Applications, vol. 389, pp. 347-353, 2004.

[36] I. Assem, D. Simson, and A. Skowroński, Elements of the Representation Theory of Associative Algebras, Volume 1, Techniques of Representation Theory, London Mathematical Society Student Texts 65, Cambridge University Press, Cambridge, UK, 2006.

[37] L. F. Barannyk, "Finite-dimensional twisted group algebras of semi-wild representation type," Colloquium Mathematicum, vol. 120, no. 2, pp. 277-298, 2010.

[38] D. Simson and A. Skowroński, Elements of the Representation Theory of Associative Algebras, Volume 2, Tubes and Concealed Algebras of Euclidean Type, London Mathematical Society Student Texts 71, Cambridge University Press, Cambridge, UK, 2007.

[39] D. Simson and M. Wojewódzki, "An algorithmic solution of a Birkhoff type problem," Fundamenta Informaticae, vol. 83, no. 4, pp. 389-410, 2008.

[40] D. Simson, "Mesh geometries of root orbits of integral quadratic forms," Journal of Pure and Applied Algebra, vol. 215, no. 1, pp. 13-34, 2011.

[41] M. Barot and J. A. de la Peña, "The Dynkin type of a nonnegative unit form," Expositiones Mathematicae, vol. 17, no. 4, pp. 339-348, 1999.

[42] M. Sato, "Periodic Coxeter matrices and their associated quadratic forms," Linear Algebra and its Applications, vol. 406, pp. 99-108, 2005. 
[43] M. Gạsiorek, D. Simson, and K. Zając, "Algorithmic computation of principal posets using Maple and Python," Algebra and Discrete Mathematics. To appear.

[44] G. Marczak, A. Polak, and D. Simson, "P-critical integral quadratic forms and positive unit forms: an algorithmic approach," Linear Algebra and its Applications, vol. 433, no. 11-12, pp. 1873-1888, 2010.

[45] D. Simson and K. Zając, "An inflation algorithm and a toroidal mesh algorithm for edge-bipartite graphs," Electronic Notes in Discrete Mathematics. To appear. 


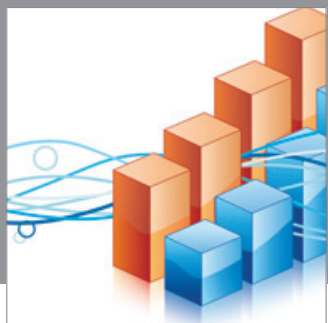

Advances in

Operations Research

mansans

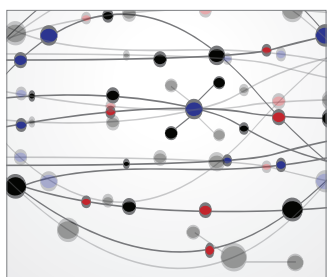

The Scientific World Journal
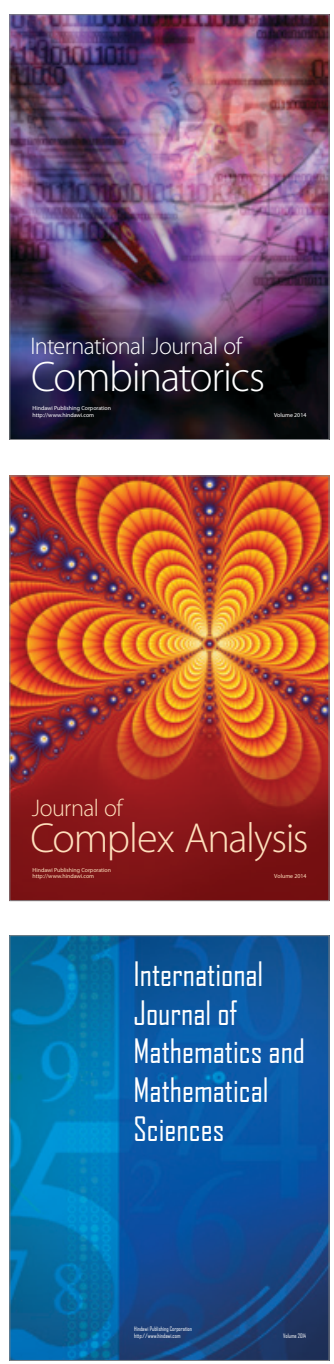
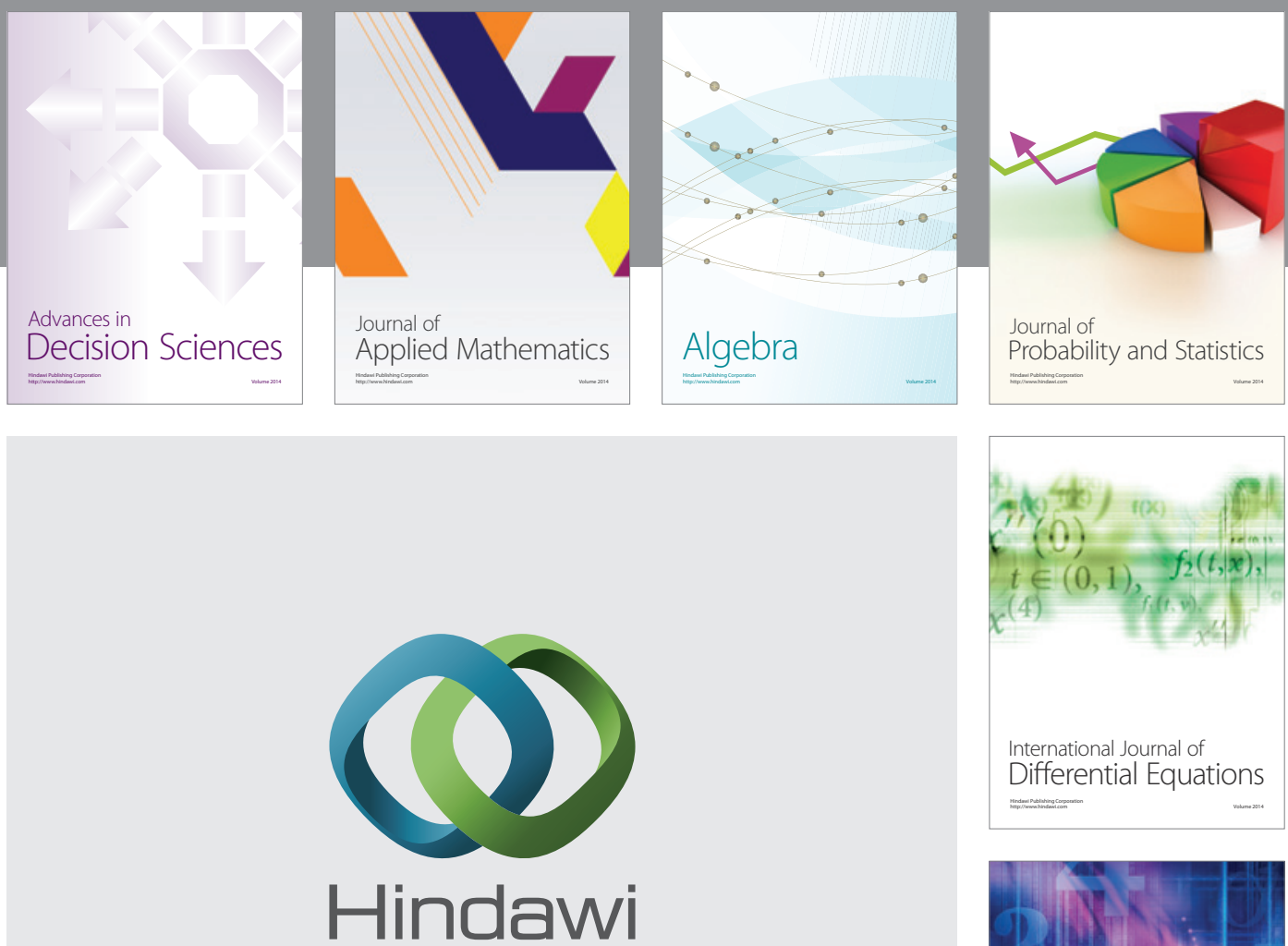

Submit your manuscripts at http://www.hindawi.com
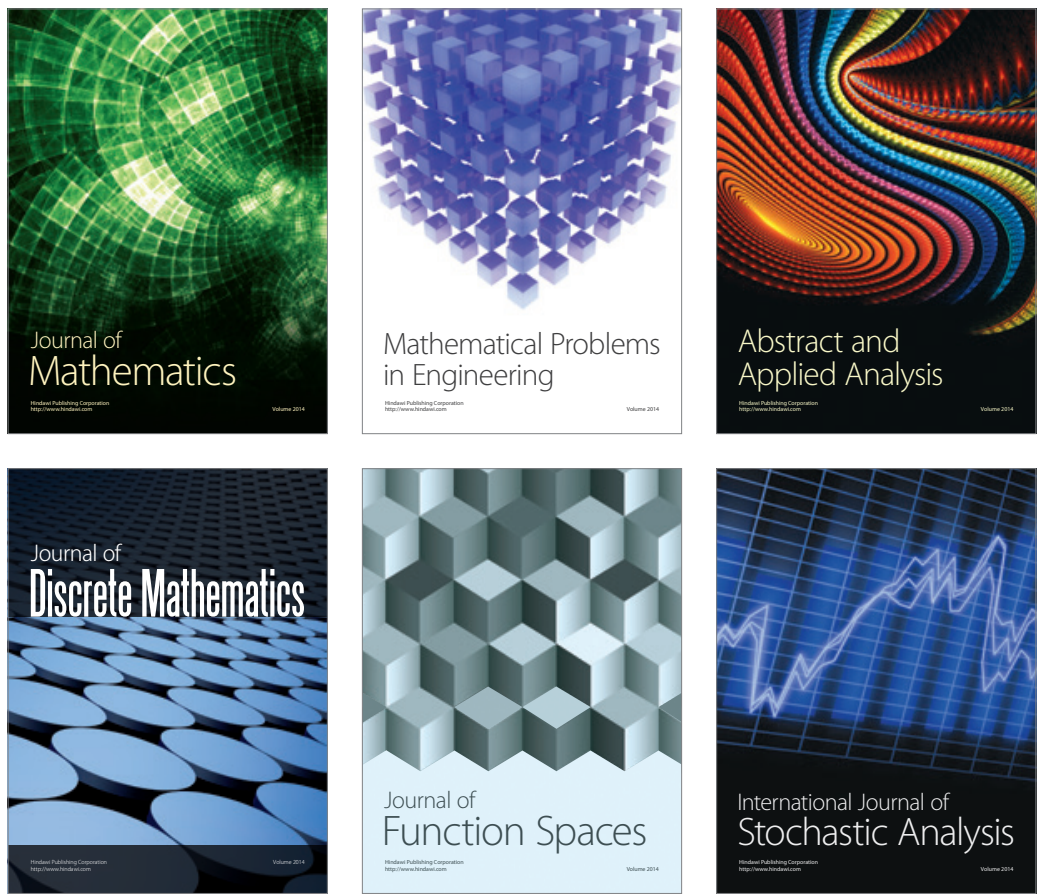

Journal of

Function Spaces

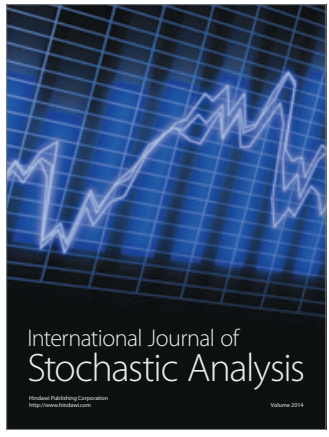

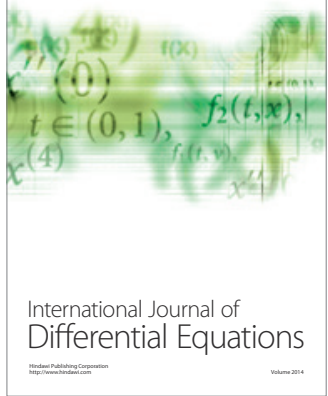
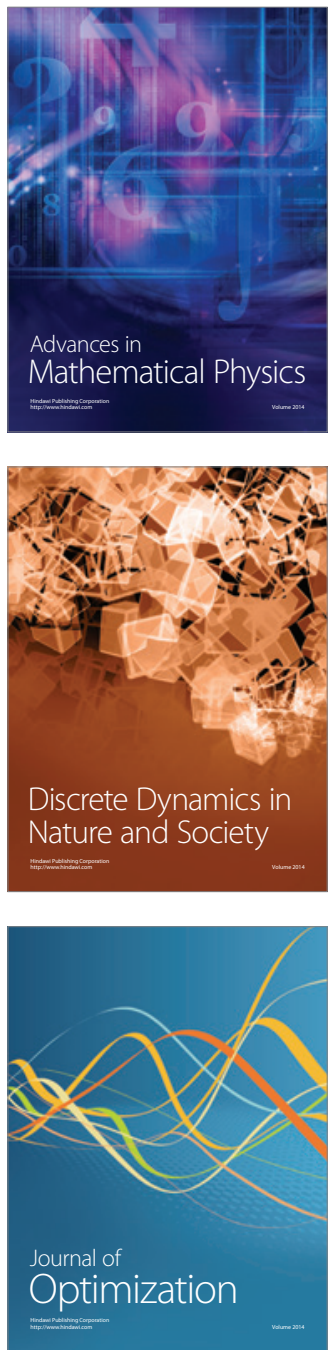